\title{
Extended analytic QCD model with perturbative QCD behavior at high momenta*
}

\author{
César Ayala ${ }^{1}$, Carlos Contreras ${ }^{1}$, and Gorazd Cvetič ${ }^{12}$ \\ ${ }^{1}$ Department of Physics, Universidad Técnica Federico Santa María (UTFSM), Valparaíso, Chile \\ ${ }^{2}$ Centro Científico-Tecnológico de Valparaíso, UTFSM, Chile
}

(Dated: July 24, 2018)

\begin{abstract}
In contrast to perturbative QCD, the analytic QCD models have running coupling whose analytic properties correctly mirror those of spacelike observables. The discontinuity (spectral) function of such running coupling is expected to agree with the perturbative case at large timelike momenta; however, at low timelike momenta it is not known. In the latter regime, we parametrize the unknown behavior of the spectral function as a sum of (two) delta functions; while the onset of the perturbative behavior of the spectral function is set to be 1.0-1.5 GeV. This is in close analogy with the "minimal hadronic ansatz" used in the literature for modeling spectral functions of correlators. For the running coupling itself, we impose the condition that it basically merges with the perturbative coupling at high spacelike momenta. In addition, we require that the well-measured nonstrange semihadronic $(V+A)$ tau decay ratio value be reproduced by the model. We thus obtain a QCD framework which is basically indistinguishable from perturbative QCD at high momenta $(Q>1 \mathrm{GeV})$, and at low momenta it respects the basic analyticity properties of spacelike observables as dictated by the general principles of the local quantum field theories.
\end{abstract}

PACS numbers: $12.38 . \mathrm{Cy}, 12.38 . \mathrm{Aw}, 12.40 . \mathrm{Vv}$

\section{INTRODUCTION}

The general principles of the local quantum field theories imply [1, 2] that the spacelike observables $\mathcal{D}\left(Q^{2}\right)$ are analytic functions of $Q^{2}$ in the complex $Q^{2}$-plane, with the nonanalyticity allowed only on the timelike semiaxis $Q^{2}<0$. Here, $-q^{2} \equiv Q^{2}$ where $q$ is the typical momentum of the considered proccess, e.g., momentum of the exchanged photon in deep inelastic scattering. However, in perturbative QCD (pQCD), the running coupling $a_{\mathrm{pt}}\left(Q^{2}\right) \equiv \alpha_{s}\left(Q^{2}\right) / \pi$ in most renormalization schemes has (Landau) singularities in the complex $Q^{2}$-plane close to the origin $\left(\left|Q^{2}\right| \lesssim 1 \mathrm{GeV}^{2}\right)$ which do not reflect the aforementioned analytic properties. This fact represents a serious, and often underestimated, conceptual and practical problem. The (spacelike) observables evaluated in pQCD, as truncated power series of $a_{\mathrm{pt}}\left(\kappa Q^{2}\right)$ (with $\kappa \sim 1$ ), do not possess the aformentioned analyticity properties; furthermore, for low values of $\left|Q^{2}\right|\left(\sim 1 \mathrm{GeV}^{2}\right)$, they are numerically unreliable due to the vicinity of the scale $\kappa Q^{2}$ to the unphysical (Landau) singularities. This numerical unreliability reflects itself in a strong dependence on the renormalization scale $(\leftrightarrow \kappa)$ and scheme.

On the other hand, studies using Dyson-Schwinger equations [3, 4] and lattice calculations [5] indicate that the QCD running coupling is finite ("conformal") at $Q^{2}=0$, and has, at least at positive $Q^{2}$ values, no Landau singularities.

The problem of Landau poles in the QCD coupling was first addressed in a systematic manner about 15 years ago by the authors of [6-8], who constructed and used an analytic QCD coupling parameter $\mathcal{A}_{1}^{(\mathrm{MA})}\left(Q^{2}\right)$ closely based on the perturbative coupling parameter $a_{\mathrm{pt}}\left(Q^{2}\right)$ : in the dispersive integral expression for $a_{\mathrm{pt}}\left(Q^{2}\right)$, they removed the integration over the offending spacelike discontinuity cut (i.e., at $-Q^{2}=\sigma<0$ ), while keeping the discontinuity (spectral) function $\rho_{1}^{(\mathrm{pt})}(\sigma) \equiv \operatorname{Im} a_{\mathrm{pt}}\left(Q^{2}=-\sigma-i \epsilon\right)$ unchanged on the timelike discontinuity cut, i.e., at $\sigma \geq 0$. Therefore, we can call this the Minimal Analytic (MA) model. The authors of [6 8 ] called it the Analytic Perturbation Approach (APT), and provided an analogous method of construction of analytic analogs $\mathcal{A}_{n}^{(\mathrm{MA})}\left(Q^{2}\right)$ of the powers $a_{\mathrm{pt}}\left(Q^{2}\right)^{n}$ for $n=2,3, \ldots$. A method of construction of analytic analogs $\mathcal{A}_{\nu}^{(\mathrm{MA})}\left(Q^{2}\right)$ of noninteger powers $a_{\mathrm{pt}}\left(Q^{2}\right)^{\nu}$ for MA (APT) was developed by the authors of [9]. Yet another analytic QCD model, based on the minimal analytization of the beta function $d a_{\mathrm{pt}}\left(Q^{2}\right) / d \ln Q^{2}$, was constructed and used in [10].

On the other hand, a more general approach of constructing analytic QCD coupling $\mathcal{A}_{1}\left(Q^{2}\right)$, based on a given choice of the discontinuity function $\rho_{1}(\sigma) \equiv \operatorname{Im} \mathcal{A}_{1}\left(Q^{2}=-\sigma-i \epsilon\right)$ (for $\sigma>0$ ) was emphasized in [11, 12] and (in a more specific context) in [13]. The spacelike coupling $\mathcal{A}_{1}\left(Q^{2}\right)$ is then constructed from $\rho_{1}(\sigma)$ by the usual dispersion relation

$$
\mathcal{A}_{1}\left(Q^{2}\right)=\frac{1}{\pi} \int_{0}^{+\infty} d \sigma \frac{\rho_{1}(\sigma)}{\left(\sigma+Q^{2}\right)}
$$

\footnotetext{
* Dedicated to Olivier Espinosa (1961-2010).
} 
From our standpoint it is this analytic coupling $\mathcal{A}_{1}$ (or equivalently: the spectral function $\rho_{1}$ ) that defines the analytic QCD (anQCD) model. The construction of analytic analogs $\mathcal{A}_{n}\left(Q^{2}\right)$ of integer powers $a_{\mathrm{pt}}\left(Q^{2}\right)^{n}$, applicable to any such analytic QCD model, was performed in [11, 12], using the relations between the logarithmic derivatives $\widetilde{a}_{\mathrm{pt}, n}\left(Q^{2}\right)$ $\left(\propto d^{n-1} a_{\mathrm{pt}}\left(Q^{2}\right) / d\left(\ln Q^{2}\right)^{n-1}\right)$ and powers $a_{\mathrm{pt}}\left(Q^{2}\right)^{k}$

$$
a_{\mathrm{pt}}^{n}=\widetilde{a}_{\mathrm{pt}, n}+\sum_{m \geq 1} \widetilde{k}_{m}(n) \widetilde{a}_{\mathrm{pt}, n+m},
$$

and $^{1}$ imposing the condition of analogy on the logarithmic derivatives of $a_{\mathrm{pt}}$ and of $\mathcal{A}_{1}: \widetilde{a}_{\mathrm{pt}, n+m} \mapsto \widetilde{\mathcal{A}}_{n+m}$. This condition was shown to be imperative, in order to keep the renormalization scale and scheme dependence of the resulting truncated analytic series (in terms of $\widetilde{\mathcal{A}}_{n}$, or $\mathcal{A}_{n}$ ) of physical observables under control. The construction of the power analogs $\mathcal{A}_{n}$ of $a_{\mathrm{pt}}^{n}$ in general analytic QCD models was obtained thus from $\mathcal{A}_{1}$ via the relations analogous to $(2)^{2}$

$$
\mathcal{A}_{n}=\widetilde{\mathcal{A}}_{n}+\sum_{m \geq 1} \widetilde{k}_{m}(n) \widetilde{\mathcal{A}}_{n+m}
$$

The extension of this construction to noninteger power analogs $\mathcal{A}_{\nu}\left(Q^{2}\right)$, for general analytic QCD models, was performed in [17].

So, from our standpoint, it remains an outstanding problem to obtain or construct the most acceptable analytic coupling $\mathcal{A}_{1}\left(Q^{2}\right)$, or equivalently, the spectral function $\rho_{1}(\sigma)=\operatorname{Im} \mathcal{A}_{1}\left(Q^{2}=-\sigma-i \epsilon\right)$ (for $\sigma>0$ ). It is reasonable to assume that at large $\sigma\left(>1 \mathrm{GeV}^{2}\right)$ we have $\rho_{1}(\sigma)=\rho_{1}^{(\mathrm{pt})}(\sigma)$, i.e., the spectral function agrees with the pQCD result. On the other hand, at low $\sigma \lesssim 1 \mathrm{GeV}^{2}$, the exact behavior of $\rho_{1}(\sigma)$ is unknown.

The construction of $\mathcal{A}_{1}\left(\widetilde{Q}^{2}\right)$ can be performed in two different ways. One way is to construct first the beta function $\beta\left(\mathcal{A}_{1}\right)=d \mathcal{A}_{1} / d \ln Q^{2}$ as function of $\mathcal{A}_{1}$. This approach is convenient if we take the position that $\beta(x)$ is an analytic function of $x$ at $x=0$. In such a case, it tuns out that we obtain perturbative QCD, i.e., $\mathcal{A}_{n}=\mathcal{A}_{1}^{n}$. However, in this case, after ensuring additionally the analyticity of $\mathcal{A}_{1}\left(Q^{2}\right)$ as a function of $Q^{2}$, it turns out to be very difficult to reproduce the correct measured value of the tau lepton (nonstrange) semihadronic $(V+A)$ decay ratio $r_{\tau} \approx 0.20$, cf. [18]. In fact, as shown in [18], the large enough value $r_{\tau}$ can be obtained in the perturbative analytic QCD frameworks only at an (unacceptable?) price of choosing a renormalization scheme with increasingly large $\beta_{j}$ coefficients, which makes the analytic perturbation series of observables convergent only when up to four terms are included, and the fifth $\left(\mathrm{N}^{4} \mathrm{LO}\right)$ term in the expansion shows an explosive increase.

Another way is to construct first the discontinuity (spectral) function $\rho_{1}(\sigma) \equiv \operatorname{Im} \mathcal{A}_{1}\left(Q^{2}=-\sigma-i \epsilon\right)$ (for $\left.\sigma>0\right)$. This approach leads, in general, to nonperturbative analytic QCD, i.e., $\mathcal{A}_{n}$ turns out to be different from $\mathcal{A}_{1}^{n}$. We can follow here analogous ideas used in the construction of the spectral functions of spacelike observables (correlators) in the literature, e.g., [19 22]. In these references, analytization is applied directly to a considered (spacelike) observable $\mathcal{D}\left(Q^{2}\right)$ itself. Some of the new nonperturbative parameters introduced there were thus specific to the chosen observable. On the other hand, we take here the standpoint that it is the (universal) QCD coupling that needs analytization; and that the additional nonperturbative contributions for a considered observable, not contained in the analytized leading-twist contribution, are accounted for by a procedure containing other universal parameters. Such parameters can be vacuum expectation values of higher dimensional operators, and the aforementioned additional nonperturbative contributions are represented by higher-twist terms of the Operator Product Expansion (OPE) [23].

In our approach, at a large enough threshold value $\sigma_{0}=M_{0}^{2}\left(\sim 1 \mathrm{GeV}^{2}\right)$ we have the onset of the perturbative behavior for the discontinuity function of the coupling

$$
\rho_{1}(\sigma)=\rho_{1}^{(\mathrm{pt})}(\sigma), \text { for } \sigma \geq M_{0}^{2}
$$

On the other hand, in the regime $0<\sigma<M_{0}^{2}$, the behavior is nonperturbative and unknown in detail, and could be parametrized as a sum (with different weights) of delta functions

$$
\rho_{1}(\sigma)=\sum_{j=1}^{n} F_{n}^{2} \delta\left(\sigma-M_{n}^{2}\right), \text { for } 0<\sigma<M_{0}^{2}
$$

\footnotetext{
1 The recurrence relations leading to the above relations, within the context of the MA (APT) model of [6- 8], were given in [14, 15].

2 The construction of higher power analogs $\mathcal{A}_{n}$ as linear operations on $\mathcal{A}_{1}$ (not as: $\mathcal{A}_{1}^{n}$ ) incorporates a nice functional property: its compatibility with linear integral transformations, such as Fourier or Laplace [16].
} 
As has been argued in 24, 25, introduction of a sufficient number of positive delta functions in the discontinuity function $\rho(\sigma)=\operatorname{Im} f\left(Q^{2}=-\sigma-i \epsilon\right)$ can approximate sufficiently well any positive Stieltjes function $f\left(Q^{2}\right)$. The analytic coupling $\mathcal{A}_{1}\left(Q^{2}\right)$ is a positive Stieltjes function [25]. On the other hand, application of such type of approximations has been applied to the spectral functions of certain current correlators [21], under the name of the "minimal hadronic ansatz."

In [26], we constructed in this way a simple one-delta analytic QCD model, by introducing one delta function in the low- $\sigma$ regime. The model contains three free parameters, ${ }^{3}$ which were fixed by the condition (two requirements) $\mathcal{A}_{1}-a_{\mathrm{pt}} \sim\left(\Lambda^{2} / Q^{2}\right)^{3}$ at large $\left|Q^{2}\right|>\Lambda^{2}$ (where $\Lambda^{2}=\Lambda_{\mathrm{QCD}}^{2} \sim 0.1 \mathrm{GeV}^{2}$ ), and the (one) requirement of reproducing the correct value of the semihadronic tau decay ratio $r_{\tau}$.

It may be regarded as overly optimistic to approximate the unknown low- $\sigma$ regime by a single delta funtion. In the present work, we go beyond the one-delta approximation, and investigate how to parametrize the low- $\sigma$ regime in a reasonable manner with two positive delta functions. Since such an extension introduces in the model several new parameters, we will fix those parameters by specific reasonable conditions, which will be similar in spirit to those of the one-delta case. Specifically, the model can be made even closer to pQCD, $\mathcal{A}_{1}-a_{\mathrm{pt}} \sim\left(\Lambda^{2} / Q^{2}\right)^{5}$ at large $\left|Q^{2}\right|>\Lambda^{2}$, while reproducing the correct value of $r_{\tau}$.

We do not introduce even more delta functions in the low- $\sigma$ regime, because in such a case we would need values of more low-energy QCD observables to fix at least some of the additional parameters. Most of the inclusive low-energy QCD observables, with the remarkable exception of the $(V+A)$ ratio $r_{\tau}$, either have large experimental uncertainties, or large theoretical uncertainties due to large higher-twist contributions, or both. On the other hand, if we fix the parameters of the model (with more than two deltas) by simply imposing a further increase in the power index $n$ of the difference $\mathcal{A}_{1}-a_{\mathrm{pt}} \sim\left(\Lambda^{2} / Q^{2}\right)^{n}(n>6)$, and without imposing the requirement of the reproduction of any additional low-energy observable value, some of the delta's become negative, indicating numerical instabilities.

In Sec. II we describe the model and impose the conditions which will fix the unknown parameters. In Sec. III we explain how to evaluate, in any anQCD model, the leading-twist contribution of the spacelike observables and of the related timelike observables, among the latter being $r_{\tau}$. In Sec. IV] we present the numerical determination of the model parameters and other numerical results. In Sec. $[\nabla$ we summarize the results of this work and outline the prospects of further applications.

\section{DESCRIPTION OF THE TWO-DELTA MODEL}

As outlined in the Introduction, we construct the two-delta (2d) anQCD model by starting with an ansatz for the discontinuity function $\rho_{1}(\sigma) \equiv \operatorname{Im} \mathcal{A}_{1}\left(Q^{2}=-\sigma-i \epsilon\right.$ ) (for $\sigma>0$ ) which agrees with the perturbative counterpart $\rho_{1}^{(\mathrm{pt})}(\sigma) \equiv \operatorname{Im} a_{\mathrm{pt}}\left(Q^{2}=-\sigma-i \epsilon\right)$ at sufficiently high scales $\sigma \geq M_{0}^{2}\left(M_{0}^{2} \sim 1 \mathrm{GeV}^{2}\right)$; and in the low-scale regime $0<\sigma<M_{0}^{2}$ its otherwise unknown behavior is parametrized as a linear combination of (two) delta functions

$$
\begin{aligned}
\rho_{1}^{(2 \mathrm{~d})}\left(\sigma ; c_{2}\right) & =\pi \sum_{j=1}^{2} f_{j}^{2} \Lambda^{2} \delta\left(\sigma-M_{j}^{2}\right)+\Theta\left(\sigma-M_{0}^{2}\right) \times \rho_{1}^{(\mathrm{pt})}\left(\sigma ; c_{2}\right) \\
& =\pi \sum_{j=1}^{2} f_{j}^{2} \delta\left(s-s_{j}\right)+\Theta\left(s-s_{0}\right) \times r_{1}^{(\mathrm{pt})}(s)
\end{aligned}
$$

where we denoted $s=\sigma / \Lambda^{2}, s_{j}=M_{j}^{2} / \Lambda^{2}(j=0,1,2)$, and $r_{1}^{(\mathrm{pt})}\left(s ; c_{2}\right)=\rho_{1}^{(\mathrm{pt})}\left(\sigma ; c_{2}\right)=\operatorname{Im} a_{\mathrm{pt}}\left(Q^{2}=-\sigma-i \epsilon ; c_{2}\right)$. Here, $\Lambda^{2}\left(\lesssim 10^{-1} \mathrm{GeV}^{2}\right)$ is the Lambert scale appearing in the following expression for $a_{\mathrm{pt}}$ :

$$
a_{\mathrm{pt}}\left(Q^{2} ; c_{2}\right)=-\frac{1}{c_{1}} \frac{1}{\left[1-c_{2} / c_{1}^{2}+W_{\mp 1}(z)\right]},
$$

where $Q^{2}=\left|Q^{2}\right| \exp (i \phi)$, the branches $W_{-1}$ and $W_{+1}$ of the Lambert function refer to the case $0 \leq \phi<+\pi$ and $-\pi<\phi<0$, respectively, ${ }^{4}$ and

$$
z=-\frac{1}{c_{1} e}\left(\frac{\left|Q^{2}\right|}{\Lambda^{2}}\right)^{-\beta_{0} / c_{1}} \exp \left(-i \beta_{0} \phi / c_{1}\right)
$$

3 In addition to the scale $\Lambda_{\mathrm{QCD}}$, which was fixed by the condition of reproducing the world average value of $a_{\mathrm{pt}}\left(M_{Z}^{2}\right)=0.119 / \pi$, in the $\overline{\mathrm{MS}}$ scheme.

4 In MATHEMATICA [27], the functions $W_{n}(z)$ are implemented by the command ProductLog $[n, z]$. 
where the aforementioned Lambert scale $\Lambda^{2}$ appears. The explicit expression (8) is the solution of the (perturbative) renormalization group equation (RGE) of the form

$$
\frac{\partial a_{\mathrm{pt}}\left(Q^{2} ; c_{2}\right)}{\partial \ln Q^{2}}=-\beta_{0} a_{\mathrm{pt}}^{2} \frac{\left[1+\left(c_{1}-\left(c_{2} / c_{1}\right)\right) a_{\mathrm{pt}}\right]}{\left[1-\left(c_{2} / c_{1}\right) a_{\mathrm{pt}}\right]} .
$$

Here, $\beta_{0}=(1 / 4)\left(11-2 n_{f} / 3\right)$ and $c_{1}=\beta_{1} / \beta_{0}=(1 / 4)\left(102-38 n_{f} / 3\right) /\left(11-2 n_{f} / 3\right)$ are the universal constants, while $c_{2}=\beta_{2} / \beta_{0}$ is the free three-loop renormalization scheme parameter. The expansion of the beta function $\beta\left(a_{\mathrm{pt}}\right)=d a_{\mathrm{pt}} / d \ln Q^{2}$ in general gives

$$
\beta\left(a_{\mathrm{pt}}\right)=-\beta_{0} a_{\mathrm{pt}}^{2}\left(1+c_{1} a_{\mathrm{pt}}+c_{2} a_{\mathrm{pt}}^{2}+c_{3} a_{\mathrm{pt}}^{3}+\ldots\right),
$$

where $c_{j}(j \geq 2)$ are general renormalization scheme parameters. The choice of the beta function on the right hand side of (10) gives $c_{j}=c_{2}^{j-1} / c_{1}^{j-2}(j \geq 3)$, which means that the three-loop scheme parameter $c_{2}$ can be chosen freely in this form, while the higher-loop scheme parameters are then fixed. The specific "effective three-loop" perturbative beta function of the Padé form of Eq. (10) was chosen for convenience, because it gives an explicit solution (8), in terms of the branches of the Lambert function $W$ [28 30], and, at the same time, it allows us to vary the renormalization scheme at the three-loop level $\left(c_{2}\right)$. In the following, it will turn out to be convenient to vary the scheme parameter $c_{2}$ in the explicit solution, the latter being used for $\rho_{1}^{(\mathrm{pt})}(\sigma) \equiv \operatorname{Im} a_{\mathrm{pt}}\left(Q^{2}=-\sigma-i \epsilon\right)$ appearing in the discontinuity function (6)-(7) of the anQCD model.

The Lambert function $W=W(z)$ is defined via the inverse relation $z=W \exp (W)$, cf. Fig. 1(a). The two branches

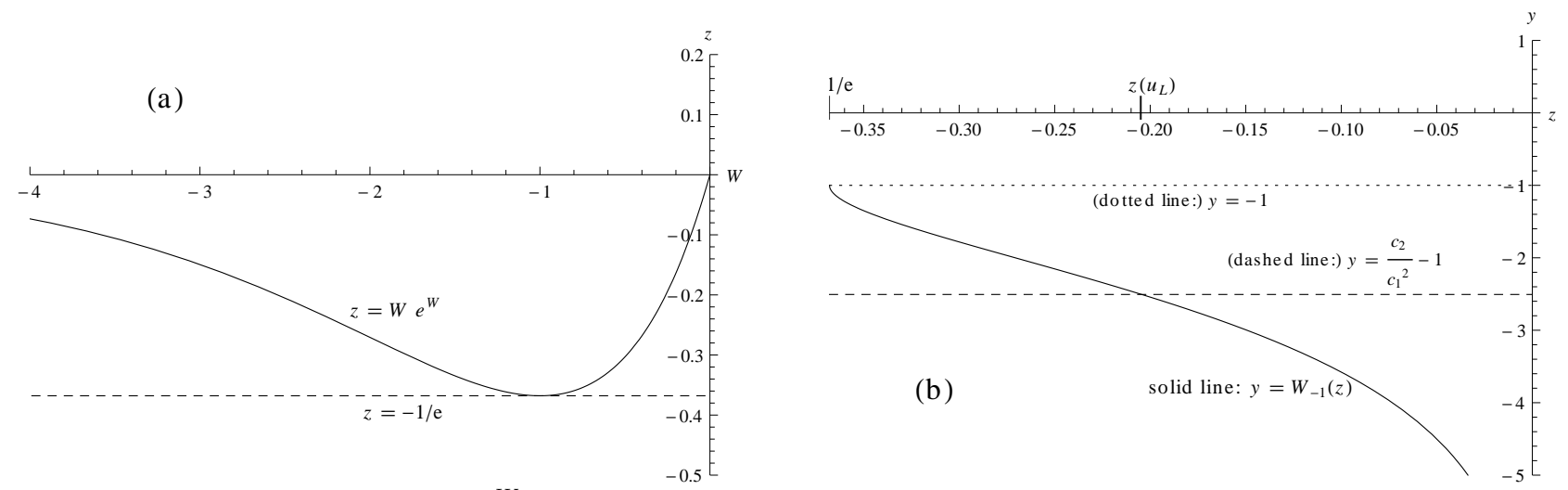

FIG. 1: (a) The defining relation $z=W e^{W}$ for the Lambert function $W(z)$, for $-1 / e<z<0$; (b) The branch $W_{-1}(z)$ for the same $z$-interval; when $c_{2}<0$, the denominator of Eq. 8 becomes zero at a $z\left(u_{\mathrm{L}}\right)$ in this interval.

$W_{\mp 1}(z)$ of the Lambert function are related via complex-conjugation $W_{+1}\left(z^{*}\right)=W_{-1}(z)^{*}$, and the point $z=-1 / e$ is the branching point of these functions. In the interval $-1 / e<z<0, W_{-1}(z)$ is a decreasing function of $z$, cf. Fig. 1(b). When $z \rightarrow-0$, the scale $Q^{2}$ tends to $Q^{2} \rightarrow+\infty$, cf. Eq. (9), and $W_{-1}(z) \rightarrow-\infty$, this reflecting the asymptotic freedom of $a_{\mathrm{pt}}\left(Q^{2}\right)$ of Eq. (8). In our considered case of low-energy QCD (i.e., with number of quark flavors $n_{f}=3$ ), the solution (8) has unphysical (Landau) singularities along the positive $Q^{2}$ axis, for any $c_{2}$. An extension of such beta function to the effective four- and five-loop case, such that the solution is explicit and involving Lambert functions, was made in [31]. For more details on the Lambert functions, we refer to Refs. 28 32].

The aforementioned branching point of nonanalyticity $z\left(s_{\mathrm{L}}\right)=-1 / e$ corresponds, according to Eq. (9), to the scale $Q^{2}\left(s_{\mathrm{L}}\right)=\Lambda^{2} s_{\mathrm{L}}$ with $s_{\mathrm{L}}=c_{1}^{-c_{1} / \beta_{0}}\left(s_{\mathrm{L}}=0.6347\right.$ when $\left.n_{f}=3\right)$, and the interval $Q^{2} \in\left(0, \Lambda^{2} s_{\mathrm{L}}\right)$ represents the interval of the unphysical (Landau) singularities of $a_{\mathrm{pt}}\left(Q^{2}\right)$ of Eq. (8). If the scheme parameter $c_{2}$ is chosen to be negative (this will be our case), then there is an additional pole-type Landau singularity at a somewhat higher scale $Q^{2}\left(u_{\mathrm{L}}\right)=\Lambda^{2} u_{\mathrm{L}}$ ( $\Leftrightarrow z=z\left(u_{\mathrm{L}}\right)=-u_{\mathrm{L}}^{-\beta_{0} / c_{1}} /\left(c_{1} e\right)$ ) at which the denominator of Eq. (8) becomes zero, cf. Fig. 1(b), i.e., when

$$
-1+c_{2} / c_{1}^{2}=W_{-1}\left(\frac{-1}{c_{1} e}\left|u_{\mathrm{L}}\right|^{-\beta_{0} / c_{1}}+i \epsilon\right) .
$$

When $n_{f}=3$ and $c_{2}=-4.76$ (this will be our central choice of the scheme later), we get $u_{\mathrm{L}}=1.0095\left(>s_{\mathrm{L}}\right)$. For this case, the coupling $a_{\mathrm{pt}}$ is presented in Fig. 2(a) as a function of $z$ (for $-1 / e<z<0$, i.e., $s_{\mathrm{L}} \Lambda^{2}<Q^{2}<\infty$ ), and in Fig. 2(b) as a function of $t=-\ln (-z)=1.266 \ln \left(Q^{2} / \Lambda^{2}\right)+1.575$. 


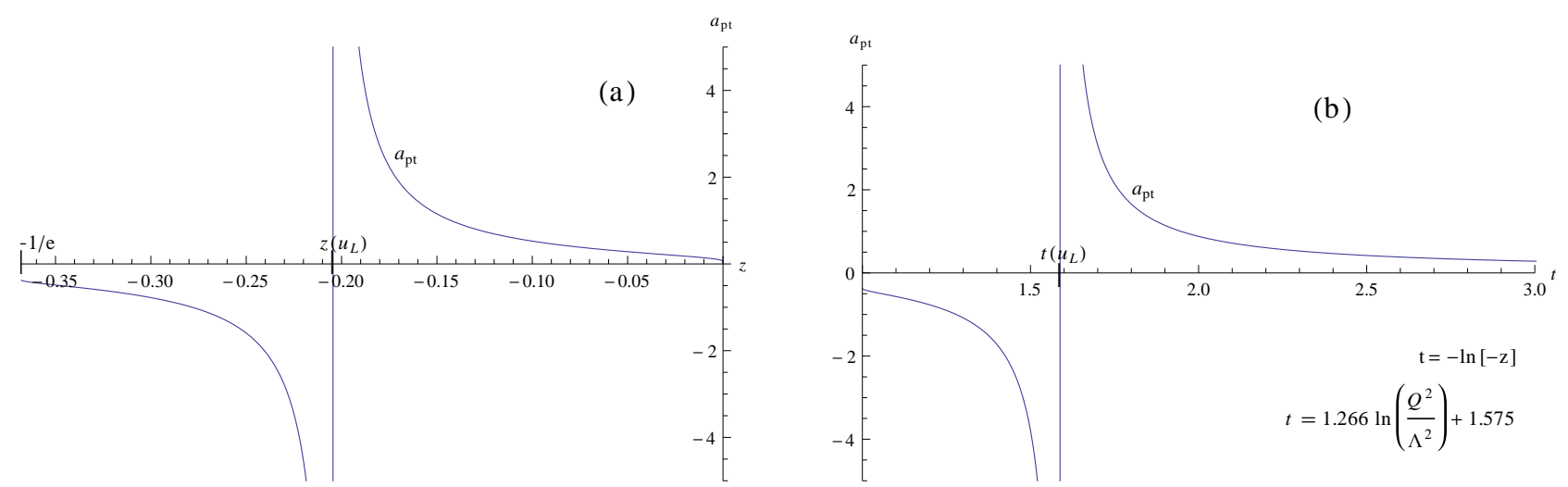

FIG. 2: (a) The perturbative coupling $a_{\mathrm{pt}}$ of Eq. (8) as a function of $z$, for $-1 / e<z<0$; (b) as a function of $t=-\ln (-z)$. The curves are for the case of $n_{f}=3$ and $c_{2}=-4.76\left(\Rightarrow t=1.266 \ln \left(Q^{2} / \Lambda^{2}\right)+1.575\right)$.

It can be checked that, as a result of application of the Cauchy theorem to the function $a_{\mathrm{pt}}\left(Q^{\prime 2}\right) /\left(Q^{\prime 2}-Q^{2}\right)$ in the complex- $Q^{\prime 2}$ plane, the following dispersion relation for $a_{\mathrm{pt}}$ holds:

$$
a_{\mathrm{pt}}\left(Q^{2} ; c_{2}\right)=\frac{1}{\pi} \int_{\sigma=-Q_{\min }^{2}-\eta^{\prime}}^{\infty} d \sigma \frac{\operatorname{Im} a_{\mathrm{pt}}\left(-\sigma-i \epsilon ; c_{2}\right)}{\left(\sigma+Q^{2}\right)}=\frac{1}{\pi} \int_{s=-u_{\min }-\eta}^{\infty} d s \frac{r_{1}^{(\mathrm{pt})}\left(s ; c_{2}\right)}{\left(s+Q^{2} / \Lambda^{2}\right)} \quad\left(\eta, \eta^{\prime} \rightarrow 0\right),
$$

where the integration covers the entire cut, i.e., starting at a sufficiently low negative value $\sigma_{\min }=-Q_{\min }^{2}\left(Q_{\min }^{2} \lesssim\right.$ $\left.1 \mathrm{GeV}^{2}\right)$. The perturbative discontinuity function is denoted as $r_{1}^{(\mathrm{pt})}\left(s ; c_{2}\right)=\operatorname{Im} a_{\mathrm{pt}}\left(Q^{2}=-s \Lambda^{2}-i \epsilon ; c_{2}\right)$. Since the cut of the coupling $a_{\mathrm{pt}}\left(Q^{\prime 2}, c_{2}\right)$, Eq. (8) with $c_{2}<0$, includes also the pole $Q_{\mathrm{L}}^{\prime 2}=u_{\mathrm{L}} \Lambda^{2}$ of the coupling, the contour of integration in the complex $\left(Q^{\prime 2} / \Lambda^{2}\right)$-plane is of the type as presented in Fig. 3 (with the outer radius going to infinity). Therefore, the dispersive relation (13) obtains a slightly generalized form

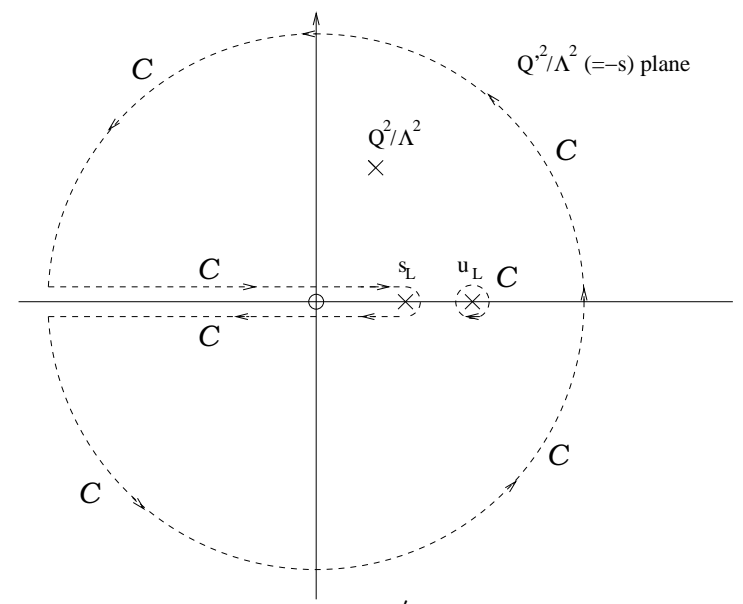

FIG. 3: The path of contour integration in $\left(Q^{\prime 2} / \Lambda^{2}\right)$-plane leading to the expression (14).

$$
\begin{aligned}
a_{\mathrm{pt}}\left(Q^{2}\right) & =\frac{1}{\pi} \int_{s=-s_{\mathrm{L}}-\eta}^{\infty} d s \frac{r_{1}^{(\mathrm{pt})}\left(s ; c_{2}\right)}{\left(s+Q^{2} / \Lambda^{2}\right)}+\frac{\Delta u}{2 \pi} \int_{\Phi=-\pi}^{\pi} d \Phi e^{i \Phi} \frac{a_{\mathrm{pt}}\left(\left(u_{\mathrm{L}}+\Delta u e^{i \Phi}\right) \Lambda^{2} ; c_{2}\right)}{\left[\left(Q^{2} / \Lambda^{2}\right)-u_{\mathrm{L}}-\Delta u e^{i \Phi}\right]} \\
& =\frac{1}{\pi} \int_{s=-s_{\mathrm{L}}-\eta}^{\infty} d s \frac{r_{1}^{(\mathrm{pt})}\left(s ; c_{2}\right)}{\left(s+Q^{2} / \Lambda^{2}\right)}+\frac{\operatorname{Res}_{\left(z=u_{\mathrm{L}}\right)} a_{\mathrm{pt}}\left(z \Lambda^{2} ; c_{2}\right)}{\left(-u_{\mathrm{L}}+Q^{2} / \Lambda^{2}\right)} .
\end{aligned}
$$

In Eq. (14), $\Delta u \Lambda^{2}$ is a sufficiently small (but otherwise arbitrary) radius of integration around the point $u_{\mathrm{L}} \Lambda^{2}$ in the complex $Q^{\prime 2}$-plane, cf. Fig. 3. In Eq. (15), this integration is expressed by the residue of the function $a_{\mathrm{pt}}\left(z \Lambda^{2}\right)$ at this point.

The perturbative discontinuity function $r_{1}^{(\mathrm{pt})}\left(s ; c_{2}\right)=\operatorname{Im} a_{\mathrm{pt}}\left(Q^{2}=-s \Lambda^{2}-i \epsilon ; c_{2}\right)$, which is nonzero for $-s_{\mathrm{L}}<s<$ 
$+\infty$, has the specific form

$$
\begin{aligned}
r_{1}^{(\mathrm{pt})}\left(s ; c_{2}\right) & =\operatorname{Im}\left[\frac{(-1)}{c_{1}} \frac{1}{\left[1-\left(c_{2} / c_{1}^{2}\right)+W_{+1}\left(\frac{-1}{c_{1} e}|s|^{-\beta_{0} / c_{1}} \exp \left(i \beta_{0} \pi / c_{1}\right)\right)\right]}\right] \quad(s>0), \\
& =\operatorname{Im}\left[\frac{(-1)}{c_{1}} \frac{1}{\left[1-\left(c_{2} / c_{1}^{2}\right)+W_{+1}\left(\frac{-1}{c_{1} e}|s|^{-\beta_{0} / c_{1}}-i \epsilon\right)\right]}\right] \quad(s<0) .
\end{aligned}
$$

The analytic (spacelike) coupling $\mathcal{A}_{1}\left(Q^{2} ; c_{2}\right)$ of the two-delta anQCD model is constructed on the basis of the discontinuity function (7) [cf. Eq. (16)] using the dispersion relation (10). This gives

$$
\mathcal{A}_{1}\left(Q^{2} ; c_{2}\right)=\sum_{j=1}^{2} \frac{f_{j}^{2}}{\left(u+s_{j}\right)}+\frac{1}{\pi} \int_{s_{0}}^{\infty} d s \frac{r_{1}^{(\mathrm{pt})}\left(s ; c_{2}\right)}{(s+u)}
$$

where $u=Q^{2} / \Lambda^{2}$.

In the presented two-delta anQCD model, we will consider the first three quark flavors to be massless, and will consider that the momenta of the $n_{f}=3$ regime in the anQCD model reach up to the threshold $\left|Q^{2}\right|=\left(2 m_{c}\right)^{2}$ $\left(\approx 6.45 \mathrm{GeV}^{2}\right)$. Further, the anQCD model will be constructed in such a way as to practically merge with pQCD at such sufficiently high momenta [in the considered renormalization scheme as fixed by Eq. (10)]. Therefore, we will consider that the value of the Lambert scale $\Lambda^{2}$ used in our analytic coupling $\mathcal{A}_{1}\left(Q^{2} ; c_{2}\right)$ coincides with the perturbative Lambert scale $\Lambda_{\mathrm{pt}}^{2}$, the latter being determined by the condition $a_{\mathrm{pt}}^{(\overline{\mathrm{MS}})}\left(M_{Z}^{2}\right)=0.1184 / \pi$, i.e., by the central value of the world average [33]. Therefore, $\Lambda^{2}$ is determined by RGE-evolving this $a_{\mathrm{pt}}$ down to $a_{\mathrm{pt}}^{(\overline{\mathrm{MS}})}\left(\left(2 m_{c}\right)^{2} ; n_{f}=3\right)$, using the four-loop polynomial form of $\beta^{(\overline{\mathrm{MS}})}\left(a_{\mathrm{pt}}\right)$, and the three-loop matching conditions 34] at quark thresholds $\mu^{2}=\left(2 m_{q}\right)^{2}(q=b, c)$; and then changing from the $\overline{\mathrm{MS}}$ scheme to the scheme ' $c_{2}$ ' $\left[\equiv\left(c_{2}, c_{3}=c_{2}^{2} / c_{1}, \ldots\right)\right]$ defined by the beta function of Eq. (10) (as explained, e.g., in Refs. [18, 26]).

The conditions we impose to fix the parameters are the following:

1. The analytic coupling $\mathcal{A}_{1}\left(Q^{2} ; c_{2}\right)$ acquires the aforementioned pQCD value of the scale $\Lambda^{2}$ of $a_{\mathrm{pt}}\left(Q^{2} ; c_{2} ; n_{f}=3\right)$ at $Q^{2}=\left(2 m_{c}\right)^{2}$

$$
\Lambda^{2}=\Lambda_{\mathrm{pt}}^{2}\left(n_{f}=3\right)
$$

2. While in general we expect $\mathcal{A}_{1}\left(Q^{2} ; c_{2}\right)$ to differ from $a_{\mathrm{pt}}\left(Q^{2} ; c_{2}\right)$ at $Q^{2}>\Lambda^{2}$ by $\sim\left(\Lambda^{2} / Q^{2}\right)^{1}$, we impose the condition

$$
\mathcal{A}_{1}\left(Q^{2} ; c_{2}\right)-a_{\mathrm{pt}}\left(Q^{2} ; c_{2}\right) \sim\left(\Lambda^{2} / Q^{2}\right)^{n_{\max }} \quad \text { with } n_{\max }=5 .
$$

The condition (20) represents in practice four conditions. In the following few lines we will describe how to formulate these four conditions.

When we subtract from the perturbative coupling (15) the analytic coupling (18), we obtain

$$
a_{\mathrm{pt}}\left(Q^{2} ; c_{2}\right)-\mathcal{A}_{1}\left(Q^{2} ; c_{2}\right)=\frac{\operatorname{Res}_{\left(z=u_{\mathrm{L}}\right)} a_{\mathrm{pt}}\left(z \Lambda^{2}\right)}{\left(u-u_{\mathrm{L}}\right)}-\sum_{j=1}^{2} \frac{f_{j}^{2}}{\left(u+s_{j}\right)}+\frac{1}{\pi} \int_{s_{\mathrm{L}}-\eta}^{s_{0}} d s \frac{r_{1}^{(\mathrm{pt})}\left(s ; c_{2}\right)}{(s+u)} .
$$

Expanding the left-hand side in powers of $(1 / u)=\left(\Lambda^{2} / Q^{2}\right)$, the imposition of the condition (20) gives us the conditions that the terms of $\sim\left(\Lambda^{2} / Q^{2}\right)^{1+k}(k=0,1,2,3$,$) in this expansion give us zero, i.e., we have the following four conditions$

$$
\frac{1}{\pi} \int_{s_{\mathrm{L}}-\eta}^{s_{0}} d s s^{k} r_{1}^{(\mathrm{pt})}\left(s ; c_{2}\right)+\left(-u_{\mathrm{L}}\right)^{k} \operatorname{Res}_{\left(z=u_{\mathrm{L}}\right)} a_{\mathrm{pt}}\left(z \Lambda^{2}\right)=s_{1}^{k} f_{1}^{2}+s_{2}^{k} f_{2}^{2} \quad(k=0,1,2,3) .
$$

Altogether, Eqs. (19) and (22) represent five conditions. Once the scheme $c_{2}$ parameter is chosen, we have altogether six parameters in the model: $f_{1}^{2}, f_{2}^{2}, s_{1}, s_{2}, s_{0}$, and the scale $\Lambda$. Therefore, yet another condition will have to be imposed, possibly involving the correct reproduction of a low-energy observable. The best candidate for this appears 
to be the canonical $(V+A)$ nonstrange and massless semihadronic $\tau$-lepton decay ratio $r_{\tau},\left[35-37 .{ }^{5}\right.$ When we remove the (measured) strangeness-changing contribution, the color and CKM factors and the electroweak effects, and the chirality-violating higher twist (quark mass) contributions, the following value is obtained (cf. 38 40], and Appendix B of Ref. [18] for details)

$$
r_{\tau}\left(\triangle S=0, m_{q}=0\right)_{\text {exp. }}=0.203 \pm 0.004 .
$$

Numerical analyses of the measured data indicate that the chirality-conserving higher-twist effects, such as gluoncondensate contributions, are negligible in the case of the considered $V+A$ decay channel. Although such analyses have been performed within pQCD+OPE approach, we will assume that they remain valid when an analysis is performed with the presented anQCD two-delta model + OPE. This assumption appears to be reasonable because the considered anQCD coupling $\mathcal{A}_{1}\left(Q^{2}\right)$ is very close to the pQCD coupling $a_{\mathrm{pt}}\left(Q^{2}\right)$ (in the considered scheme) at momenta $\left|Q^{2}\right| \gtrsim 1 \mathrm{GeV}^{2}$. Therefore, in the calculation of the discussed $r_{\tau}$, Eq. (23), in the presented anQCD model, the (chirality-conserving) higher-twist contributions will be ignored.

\section{CALCULATION OF ADLER FUNCTION AND $r_{\tau}$ IN ANALYTIC QCD}

The calculation of $r_{\tau}$ is then performed in the same way as presented in [18, 26], i.e., by performing explicitly the integration corresponding to the leading- $\beta_{0}(\mathrm{LB})$ resummation for $r_{\tau}$, and adding the three known beyond-LB (bLB) terms (i.e., including the exact contributions of $\sim \mathcal{A}_{4}$ ).

In this Section we will present only the main points of calculation of spacelike (such as Adler function) and timelike quantities (such as $r_{\tau}$ ) in anQCD models. For details, we refer to [11, 12, 26], and especially Appendices C and D of [18].

The basic idea of the approach in the evaluation of spacelike observables $\mathcal{D}\left(Q^{2}\right)$ in general anQCD model is to reorganize first the perturbation series $\mathcal{D}\left(Q^{2}\right)_{\mathrm{pt}}$

$$
\mathcal{D}\left(Q^{2}\right)_{\mathrm{pt}}=a_{\mathrm{pt}}+d_{1} a_{\mathrm{pt}}^{2}+d_{2} a_{\mathrm{pt}}^{3}+\ldots,
$$

into the modified perturbation series (mpt)

$$
\mathcal{D}\left(Q^{2}\right)_{\mathrm{mpt}}=a_{\mathrm{pt}}+\widetilde{d}_{1} \widetilde{a}_{\mathrm{pt}, 2}+\widetilde{d}_{2} \widetilde{a}_{\mathrm{pt}, 3}+\ldots,
$$

where $\widetilde{d}_{k} \widetilde{a}_{\mathrm{pt}, k+1}$ are the logarithmic derivatives of $a_{\mathrm{pt}}$

$$
\widetilde{a}_{\mathrm{pt}, k+1}\left(Q^{2}\right) \equiv \frac{(-1)^{k}}{\beta_{0}^{k} k !} \frac{\partial^{k} a_{\mathrm{pt}}\left(Q^{2}\right)}{\partial\left(\ln Q^{2}\right)^{k}}, \quad(k=0,1,2, \ldots),
$$

They are related with the powers of $a_{\mathrm{pt}} \equiv \alpha_{s} / \pi$ in the following way [using renormalization group equations (RGE) in pQCD]:

$$
\begin{aligned}
& \widetilde{a}_{\mathrm{pt}, 2}=a_{\mathrm{pt}}^{2}+c_{1} a_{\mathrm{pt}}^{3}+c_{2} a_{\mathrm{pt}}^{4}+\ldots, \\
& \widetilde{a}_{\mathrm{pt}, 3}=a_{\mathrm{pt}}^{3}+\frac{5}{2} c_{1} a_{\mathrm{pt}}^{4}+\ldots, \quad \widetilde{a}_{\mathrm{pt}, 4}=a_{\mathrm{pt}}^{4}+\ldots, \quad \text { etc. }
\end{aligned}
$$

This, in turn, means that the powers of $a_{\mathrm{pt}}$ are linear combinations of logarithmic derivatives

$$
\begin{aligned}
& a_{\mathrm{pt}}^{2}=\widetilde{a}_{\mathrm{pt}, 2}-c_{1} \widetilde{a}_{\mathrm{pt}, 3}+\left(\frac{5}{2} c_{1}^{2}-c_{2}\right) \tilde{a}_{\mathrm{pt}, 4}+\ldots, \\
& a_{\mathrm{pt}}^{3}=\widetilde{a}_{\mathrm{pt}, 3}-\frac{5}{2} c_{1} \widetilde{a}_{\mathrm{pt}, 4}+\ldots, \quad a_{\mathrm{pt}}^{4}=\widetilde{a}_{\mathrm{pt}, 4}+\ldots, \quad \text { etc. }
\end{aligned}
$$

which allows us to relate the mpt coefficients with the usual perturbation series (pt) coefficients

$$
\begin{aligned}
& \widetilde{d}_{1}=d_{1}, \quad \widetilde{d}_{2}=d_{2}-c_{1} d_{1}, \\
& \widetilde{d}_{3}=d_{3}-\frac{5}{2} c_{1} d_{2}+\left(\frac{5}{2} c_{1}^{2}-c_{2}\right) d_{1}, \quad \text { etc. }
\end{aligned}
$$

5 The "canonical" means that the normalization is used such that $\left(r_{\tau}\right)_{\mathrm{pt}}=a_{\mathrm{pt}}+\mathcal{O}\left(a_{\mathrm{pt}}^{2}\right)$. 
In [11, 12] it was shown that it is imperative to construct first the analogs of the logarithmic derivatives of $a_{\mathrm{pt}}$ in the following way: ${ }^{6}$

$$
\left(\frac{\partial^{k} a_{\mathrm{pt}}\left(Q^{2}\right)}{\partial\left(\ln Q^{2}\right)^{k}}\right)_{\mathrm{an}}=\frac{\partial^{k}}{\partial\left(\ln Q^{2}\right)^{k}}\left(a_{\mathrm{pt}}\left(Q^{2}\right)\right)_{\mathrm{an}}=\frac{\partial^{k} \mathcal{A}_{1}\left(Q^{2}\right)}{\partial\left(\ln Q^{2}\right)^{k}} \quad(k=0,1,2, \ldots) .
$$

This means that the 'mpt' expansion (25) becomes in anQCD the corresponding "modified analytic series" ('man')

$$
\mathcal{D}\left(Q^{2}\right)_{\operatorname{man}}=\mathcal{A}_{1}+\widetilde{d}_{1} \widetilde{\mathcal{A}}_{2}+\widetilde{d}_{2} \widetilde{\mathcal{A}}_{3}+\ldots
$$

and its truncated version is

$$
\mathcal{D}\left(Q^{2}\right)_{\text {man }}^{[N]}=\mathcal{A}_{1}+\widetilde{d}_{1} \widetilde{\mathcal{A}}_{2}+\ldots+\widetilde{d}_{N-1} \widetilde{\mathcal{A}}_{N}
$$

Here we denoted by $\widetilde{\mathcal{A}}_{k+1}$ the logarithmic derivatives of $\mathcal{A}_{1}$

$$
\widetilde{\mathcal{A}}_{k+1}\left(\mu^{2}\right)=\frac{(-1)^{k}}{\beta_{0}^{k} k !} \frac{\partial^{k} \mathcal{A}_{1}\left(\mu^{2}\right)}{\partial\left(\ln \mu^{2}\right)^{k}}, \quad(k=1,2, \ldots) .
$$

The expressions (34)-(36) are the basis of the evaluation of massless spacelike observables in any anQCD. ${ }^{7}$

The quantity $r_{\tau}$ is, on the other hand, a timelike observable. However, it is expressed via a contour integration [41]

$$
r_{\tau}=\frac{1}{2 \pi} \int_{-\pi}^{+\pi} d \phi\left(1+e^{i \phi}\right)^{3}\left(1-e^{i \phi}\right) d_{\mathrm{Adl}}\left(Q^{2}=m_{\tau}^{2} e^{i \phi}\right)
$$

which involves the (spacelike, massless, $n_{f}=3$ ) Adler function $d_{\mathrm{Adl}}\left(Q^{2}\right)=a_{\mathrm{pt}}\left(Q^{2}\right)+\mathcal{O}\left(a_{\mathrm{pt}}^{2}\right)$. The perturbation expansion of $d_{\mathrm{Adl}}$ is known up to $\sim a_{\mathrm{pt}}^{4}[42,44]$

$$
d_{\mathrm{Adl}}\left(Q^{2}\right)_{\mathrm{pt}}^{[4]}=a_{\mathrm{pt}}+\sum_{n=1}^{3}\left(d_{\mathrm{Adl}}\right)_{n} a_{\mathrm{pt}}^{n+1}
$$

On the other hand, the leading- $\beta_{0}$ parts $\left(d_{\mathrm{Adl}}\right)_{n}^{(\mathrm{LB})}=c_{n, n}^{(1)} \beta_{0}^{n}\left[=\left(\widetilde{d}_{\mathrm{Adl}}\right)_{n}^{(\mathrm{LB})}\right]$ of all the coefficients $\left(d_{\mathrm{Adl}}\right)_{n}$ are known ${ }^{8}$ [45, 46] and the resummation of these contributions can be performed by using the approach of Neubert of integration with characteristic functions [47] - this can be performed in any anQCD without ambiguities (since no Landau singularities)

$$
\begin{aligned}
\left(d_{\mathrm{Adl}}\right)_{\operatorname{man}}^{(\mathrm{LB})}\left(Q^{2}\right) & \equiv \mathcal{A}_{1}\left(Q^{2}\right)+c_{1,1}^{(1)} \beta_{0} \widetilde{\mathcal{A}}_{2}\left(Q^{2}\right)+\ldots+c_{n, n}^{(1)} \beta_{0}^{n} \widetilde{\mathcal{A}}_{n+1}\left(Q^{2}\right)+\ldots \\
& =\int_{0}^{\infty} \frac{d t}{t} F_{\mathrm{Adl}}(t) \mathcal{A}_{1}\left(t Q^{2} e^{-5 / 3}\right) .
\end{aligned}
$$

Here, $F_{\text {Adl }}(t)$ is the characteristic function of the Adler function, whose explicit expression was obtained in [47].

On the other hand, the full coefficients $\left(d_{\mathrm{Adl}}\right)_{n}$ and $\left(\widetilde{d}_{\mathrm{Adl}}\right)_{n}$ are known only up to $n=3$ [2 44 . Therefore, the full Adler function can be evaluated in anQCD by adding to the leading- $\beta_{0}$ (LB) contribution the three known beyond-LB (bLB) terms

$$
\left(d_{\mathrm{Adl}}\right)_{\operatorname{man}}^{(\mathrm{LB}+\mathrm{bLB})}\left(Q^{2}\right)^{[4]}=\int_{0}^{\infty} \frac{d t}{t} F_{\mathrm{Adl}}^{\mathcal{E}}(t) \mathcal{A}_{1}\left(t Q^{2} e^{-5 / 3}\right)+\sum_{n=1}^{3}\left(T_{\mathrm{Adl}}\right)_{n} \widetilde{\mathcal{A}}_{n+1}
$$

6 If the analytization is performed in any other way, the renormalization scale and scheme dependence of the resulting truncated analytic series of any observable $\mathcal{D}\left(Q^{2}\right)$ will in general increase (instead of decrease) when the number of terms in the series increases, cf. [11, 12].

7 If masses are involved in the evaluated physical quantity, perturbation series contains noninteger powers $a_{\mathrm{pt}}^{\nu}$ (and possibly derivatives thereof with respect to $\nu$, i.e., $\left.a_{\mathrm{pt}}^{\nu} l n^{k} a_{\mathrm{pt}}\right)$. The evaluation of such quantities in anQCD models is then based on the procedure presented in [17].

${ }^{8}$ We have $\left(d_{\mathrm{Adl}}\right)_{n}=c_{n, n}^{(1)} \beta_{0}^{n}+\mathcal{O}\left(\beta_{0}^{n-1}\right)$ and $\left(\widetilde{d}_{\mathrm{Adl}}\right)_{n}=c_{n, n}^{(1)} \beta_{0}^{n}+\mathcal{O}\left(\beta_{0}^{n-1}\right)$. The expansions in powers of $\beta_{0}$ are obtained when $\left(d_{\mathrm{Adl}}\right)_{n}$ and $\left(\widetilde{d}_{\mathrm{Adl}}\right)_{n}$ are written in powers of $n_{f}\left(=-6 \beta_{0}+33 / 2\right)$ and then reorganized in powers of $\beta_{0}$. 
where

$$
\left(T_{\mathrm{Adl}}\right)_{n}=\left(\widetilde{d}_{\mathrm{Adl}}\right)_{n}-c_{n n}^{(1)} \beta_{0}^{n}
$$

are the complete bLB parts $\left(\sim \beta_{0}^{n-1}\right)$ of the coefficients $\left(\widetilde{d}_{\mathrm{Adl}}\right)_{n}$.

Using the expression (41) in the contour integration (37) gives for $r_{\tau}$

$$
r_{\tau}^{(\mathrm{LB}+\mathrm{bLB}),[4]}=r_{\tau}^{(\mathrm{LB})}+\sum_{n=1}^{3}\left(T_{\mathrm{Adl}}\right)_{n} I\left(\widetilde{\mathcal{A}}_{n+1}\right),
$$

where $I\left(\widetilde{\mathcal{A}}_{n+1}\right)$ are the contour integrals given by

$$
I\left(\widetilde{\mathcal{A}}_{n+1}\right)=\frac{1}{2 \pi} \int_{-\pi}^{+\pi} d \phi\left(1+e^{i \phi}\right)^{3}\left(1-e^{i \phi}\right) \widetilde{\mathcal{A}}_{n+1}\left(m_{\tau}^{2} e^{i \phi}\right) \quad(n=1,2,3),
$$

and the LB part in (43) is a well-defined (in anQCD models) integral of the form

$$
r_{\tau}^{(\mathrm{LB})}=\frac{1}{\pi} \int_{0}^{\infty} \frac{d t}{t} \widetilde{F}_{r}(t) \rho_{1}\left(\mathrm{tm}_{\tau}^{2} e^{-5 / 3}\right),
$$

where $\widetilde{F}_{r}(t)$ was calculated in [18] from the Minkowskian characteristic function $F_{r}^{\mathcal{M}}(t)$ of [48]. For details on $\widetilde{F}_{r}(t)$ we refer to App. D of [18]..$^{9}$

Thus, the more explicit form of the $r_{\tau}$-reproduction condition, mentioned in the previous section, is

$$
\left(r_{\tau}^{(\mathrm{LB}+\mathrm{bLB}[4])}=\right) r_{\tau}^{(\mathrm{LB})}+\sum_{n=1}^{3}\left(T_{\mathrm{Adl}}\right)_{n} I\left(\widetilde{\mathcal{A}}_{n+1}\right)=0.203 .
$$

The six conditions, (19), (22) and (46), then determine the six parameters of the model: $f_{j}^{2}, f_{2}^{2}, s_{1}, s_{2}, s_{0}$ and the scale $\Lambda$. This procedure can be performed once we have chosen a value of the scheme parameter $c_{2}$ of Eq. (10).

\section{NUMERICAL RESULTS}

In this Section we present the numerical results for the parameters of the model, obtained from the imposition of the aforementioned six conditions. The additional (implicit) conditions that we choose are that the weights $f_{1}^{2}$ and $f_{2}^{2}$ are positive. This is based on the fact that the discontinuity function $\rho_{1}(\sigma)$ is positive in any reasonable scheme of pQCD. Furthermore, the condition of positivity of $\rho_{1}(\sigma)$ can be expressed also via the condition that the Minkowskian coupling

$$
\mathfrak{A}_{1}(\sigma)=\frac{1}{\pi} \int_{\sigma}^{\infty} d \sigma^{\prime} \frac{\rho_{1}\left(\sigma^{\prime}\right)}{\sigma^{\prime}}
$$

is a monotonously decreasing function of scale $\sigma$.

If we choose for the scheme parameter $c_{2}=0$, we obtain from the six conditions, as a result, that the pQCD-onset mass $M_{0}=\sqrt{s_{0}} \Lambda$ is relatively high, $M_{0} \approx 1.7 \mathrm{GeV}$. Increasing $c_{2}$ to positive values increases $M_{0}$ further; e.g., for $c_{2}=c_{2}^{\overline{\mathrm{MS}}} / 2(\approx 2.24)$ we get $M_{0} \approx 2.0 \mathrm{GeV}$, etc. On the other hand, decreasing $c_{2}$ to negative values, we obtain smaller $M_{0}$. We believe that the effective pQCD-onset scale $M_{0}$ should be significantly smaller than the mass of the $\tau$ lepton. This turned out to be so in the one-delta anQCD model of [26], with $c_{2}=0$, where $M_{0} \approx 1 \mathrm{GeV}$ was obtained. In general, we do not want to parametrize (via delta functions) relatively small deviations from pQCD, i.e., those at $\sqrt{\sigma}>1.5 \mathrm{GeV}$. On the other hand, by lowering the value of $c_{2}$, we encounter at $c_{2}<-8.0\left(M_{0}<0.9 \mathrm{GeV}\right)$ negative values of $s_{2}$, implying that the analyticity is lost.

Therefore, we will adjust the scheme parameter $c_{2}$ in such a way as to get $M_{0}=1.25 \pm 0.25 \mathrm{GeV}$. The results are given in Table I. We believe that all three choices of $M_{0}$ will give almost the same predictions for various physical

9 In (43)- (44) we used in the Adler function the renormalization scale $\mu^{2}=Q^{2}\left(\equiv m_{\tau}^{2} e^{i \phi}\right)$; this scale, of course, can be varied, cf. [18, 26] for details. 
TABLE I: The parameters of the considered two-delta anQCD model, for the three chosen values of the pQCD-onset scale $M_{0}$ : $1.0 ; 1.25 ; 1.50 \mathrm{GeV}$. In addition, the results of the one-delta (1d) model of [26] in the scheme $c_{2}=0$ are given (the last line). See the text for details.

\begin{tabular}{l|lllllll}
\hline$M_{0}[\mathrm{GeV}]$ & $c_{2}=\beta_{2} / \beta_{0}$ & $\Lambda[\mathrm{GeV}]$ & $s_{0}$ & $s_{1}$ & $f_{1}^{2}$ & $s_{2}$ & $f_{2}^{2}$ \\
\hline 1.00 & -7.15 & 0.193 & 26.86 & 19.473 & 0.3637 & 0.3594 & 0.7808 \\
1.25 & -4.76 & 0.260 & 23.06 & 16.837 & 0.2713 & 0.8077 & 0.5409 \\
1.50 & -2.10 & 0.363 & 17.09 & 12.523 & 0.1815 & 0.7796 & 0.3462 \\
\hline $0.886(1 \mathrm{~d})$ & 0 & 0.472 & 3.525 & 0.4755 & 0.2086 & $\cdots$ & 0.5476 \\
\hline \hline
\end{tabular}

observables. The reason for this lies in the renormalization scheme independence of pQCD results; and our model, although nonperturbative and analytic at low momenta $|Q| \lesssim 1 \mathrm{GeV}$, is practically indistinguishable from pQCD at all higher momenta. It is interesting that the value of the coupling $\mathcal{A}_{1}$ at $Q^{2}=0$, obtained in this model for $M_{0}=1.25 \mathrm{GeV}\left(\mathcal{A}_{1}(0) \approx 0.8\right)$ is not far from the value in Ref. $[3]\left(\mathcal{A}_{1}(0) \approx 8.9 / N_{c} / \pi \approx 0.9\right)$, which was obtained from an analysis using Dyson-Schwinger equations for the ghost and gluon sector under an assumption of regularity of the ghost-gluon vertex.

In Table \we included, in the last line, also the results of the one-delta (1d) model of [26], obtained in an analogous way, in the scheme $c_{2}=c_{3}=\ldots=0 .{ }^{10}$ In that model, though, the smaller number of parameters led to less stringent conditions (20), namaly with $n_{\max }=3$ (cf. also a similar model in [50]).

In Fig. 4(a), we present, for the resulting "central" choice of $M_{0}=1.25 \mathrm{GeV}\left(c_{2}=-4.76\right)$, the corresponding pQCD discontinuity function $\rho_{1}^{(\mathrm{pt})}(\sigma)=\operatorname{Im} a_{\mathrm{pt}}(-\sigma-i \epsilon)$ of the underlying perturbative coupling $a_{\mathrm{pt}}$ (8), in the regime of low $|\sigma|$ and including the unphysical (Landau) regime of negative- $\sigma$ cut. We can note that in the latter regime there is an additional, pole-like singularity at $\sigma=-u_{\mathrm{L}} \Lambda^{2}\left(\approx-0.261 \mathrm{GeV}^{2}\right)$; while the "continuous" part of $\rho_{1}^{(\mathrm{pt})}(\sigma)$ ends a bit
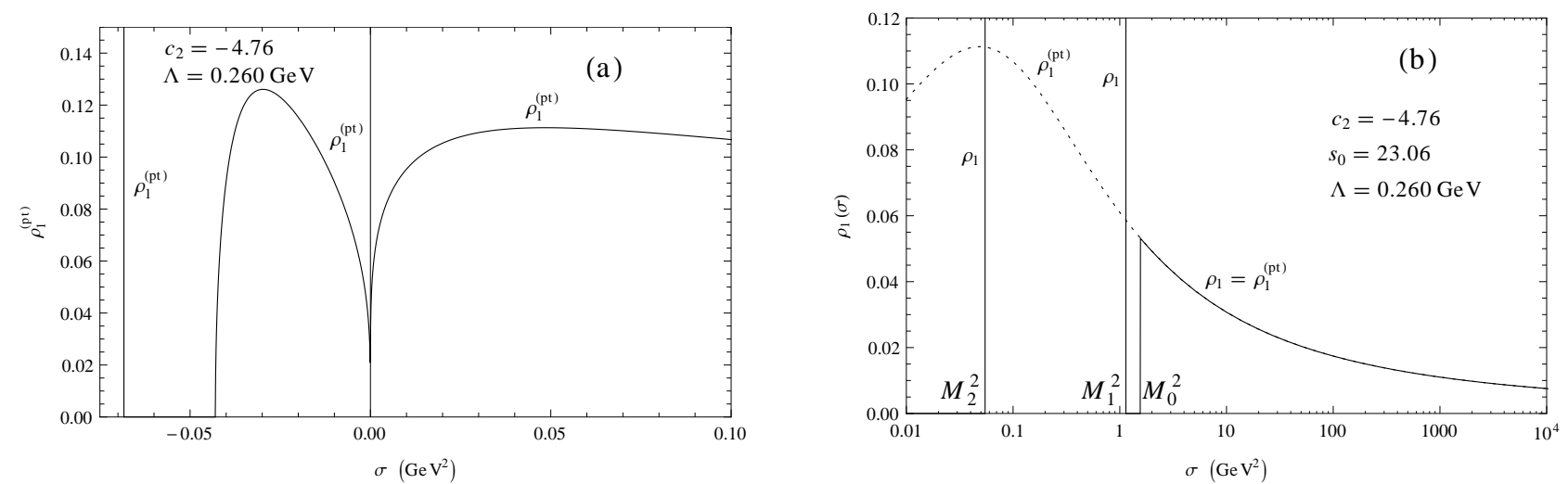

FIG. 4: The discontinuity function $\rho_{1}(\sigma)$ : (a) for the underlying pQCD coupling $a_{\mathrm{pt}}\left(Q^{2}\right)$, where the Landau pole at $\sigma=-u_{\mathrm{L}} \Lambda^{2}$ $\left(\approx-0.068 \mathrm{GeV}^{2}\right)$ and the branching point at $\sigma=-s_{\mathrm{L}} \Lambda^{2}\left(\approx-0.043 \mathrm{GeV}^{2}\right)$ are visible; (b) for the considered two-delta anQCD model, where the unknown region $0<\sigma<M_{0}^{2}$ is parametrized by two delta functions, at $\sigma=M_{1}^{2}$ and $\sigma=M_{2}^{2}$. The parameters used correspond to the central case in Table $1\left(M_{0}=1.25 \mathrm{GeV}\right)$.

earlier, at $\sigma=-s_{\mathrm{L}} \Lambda^{2} \approx-0.207 \mathrm{GeV}^{2} .{ }^{11}$ In Fig. $4(\mathrm{~b}), \rho_{1}(\sigma)$ of the considered two-delta anQCD model is presented, cf Eq. (6).

In Figs. [5(a) and (b), we present, for the aforementioned central case $M_{0}=1.25 \mathrm{GeV}$, the resulting spacelike coupling $\mathcal{A}_{1}\left(Q^{2}\right)$ at positive $Q^{2}$. The higher order couplings $\widetilde{\mathcal{A}}_{k}\left(Q^{2}\right)(k=2,3)$, cf. Eq. (36), are also presented; due to a strong hierarchy (even at low $Q^{2}$ ) they are rescaled, for better visibility, by factors 4 and 16, respectively. All the corresponding pQCD quantities $\left(a_{\mathrm{pt}}, 4 \widetilde{a}_{\mathrm{pt}, 2}, 16 \widetilde{a}_{\mathrm{pt}, 3}\right)$ are presented as dotted curves. For better visibility at low $Q^{2}$, Fig. 5 (b) is presented with $Q^{2}$ on logarithmic scale. It is clearly visible that the model practically agrees with the

10 In Ref. [26], the parameters differ a little from those in Table I because the world average value taken there was from the year $2008, a_{\mathrm{pt}}\left(M_{Z}^{2} ; \overline{\mathrm{MS}}\right)=0.1190 / \pi,[49]$; and because we imposed there the condition $\mathcal{A}_{1}\left(\left(3 m_{c}\right)^{2}\right)=a_{\mathrm{pt}}\left(\left(3 m_{c}\right)^{2} ; n_{f}=3\right)$ instead of the (numerically similar) condition (19).

11 Note: $s_{\mathrm{L}}=c_{1}^{-c_{1} / \beta_{0}}$, which is approximately 0.635 when $n_{f}=3$; at $Q^{2}=s_{\mathrm{L}} \Lambda^{2}$ the Lambert function $W_{-1}\left(z\left(Q^{2}\right)\right)$ is equal to -1 , and this is the branching point for $a_{\mathrm{pt}}\left(Q^{2}\right)$, cf. [28, 31]. 

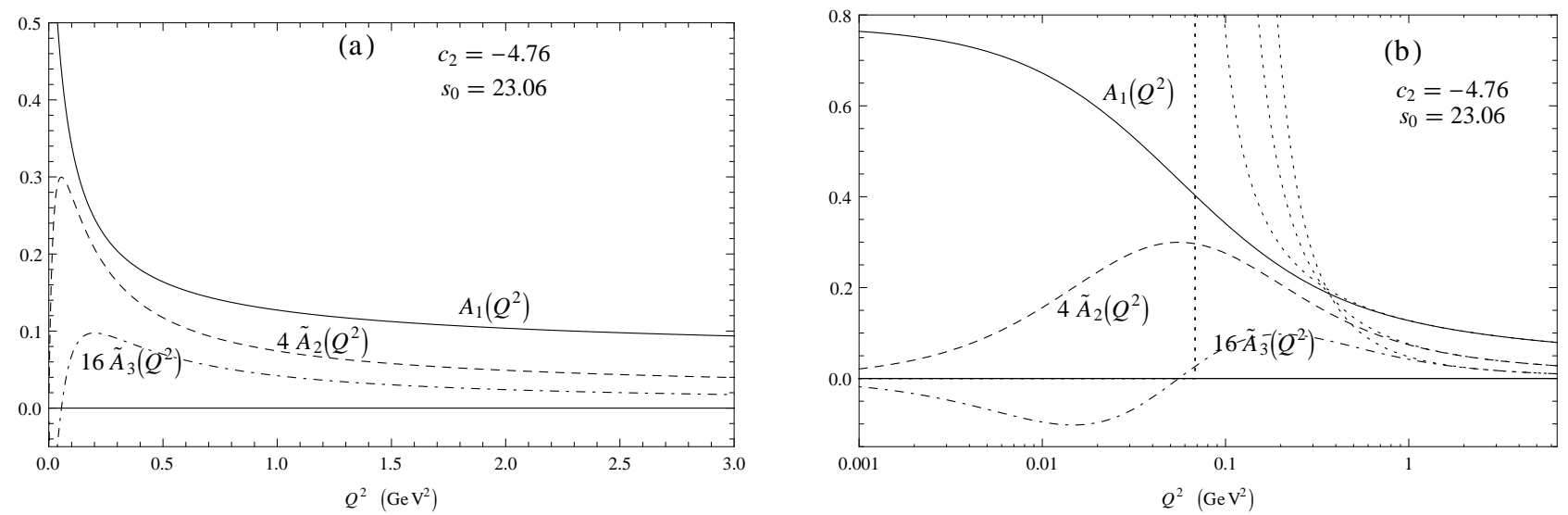

FIG. 5: The analytic couplings $\mathcal{A}_{1}$ (full line), $4 \times \widetilde{\mathcal{A}}_{2}$ (dashed curve), and $16 \times \widetilde{\mathcal{A}}_{3}$ (dash-dotted curve) for positive $Q^{2}$, in the considered two-delta model, for the central parameter choice of Table \ $\left(M_{0}=1.25 \mathrm{GeV}\right)$ : (a) linear scale is used for $Q^{2}$; (b) logarithmic scale is used for $Q^{2}$, for better visibility at low values of $Q^{2}$. For comparison, the corresponding pQCD couplings $\left(a_{\mathrm{pt}}, 4 \times \widetilde{a}_{\mathrm{pt}, 2}, 16 \times \widetilde{a}_{\mathrm{pt}, 3}\right)$ are included, as dotted curves (in the same renormalization scheme, with $c_{2}=-4.76$ ); the vertical dotted line is the Landau pole at $Q^{2}=u_{\mathrm{L}} \Lambda^{2}\left(\approx 0.068 \mathrm{GeV}^{2}\right)$.

corresponding pQCD model at $Q^{2}>1 \mathrm{GeV}^{2}$; and that for $Q^{2}<1 \mathrm{GeV}^{2}$ the model differs from pQCD significantly, due to the imposition of the analyticity.

In Fig. 66(a), we present the difference between the perturbative and the analytic coupling $\left(a_{\mathrm{pt}}\left(Q^{2}\right)-\mathcal{A}_{1}\left(Q^{2}\right)\right)$ at positive $Q^{2}$, for the central choice of parameter $\left(M_{0}=1.25 \mathrm{GeV}\right)$ of Table \. In the Figure we keep, formally, $n_{f}=3$ even for high $Q^{2}$. We see that the difference vanishes fast when $Q^{2}$ increases. For comparison, in Fig. 6(b) we show
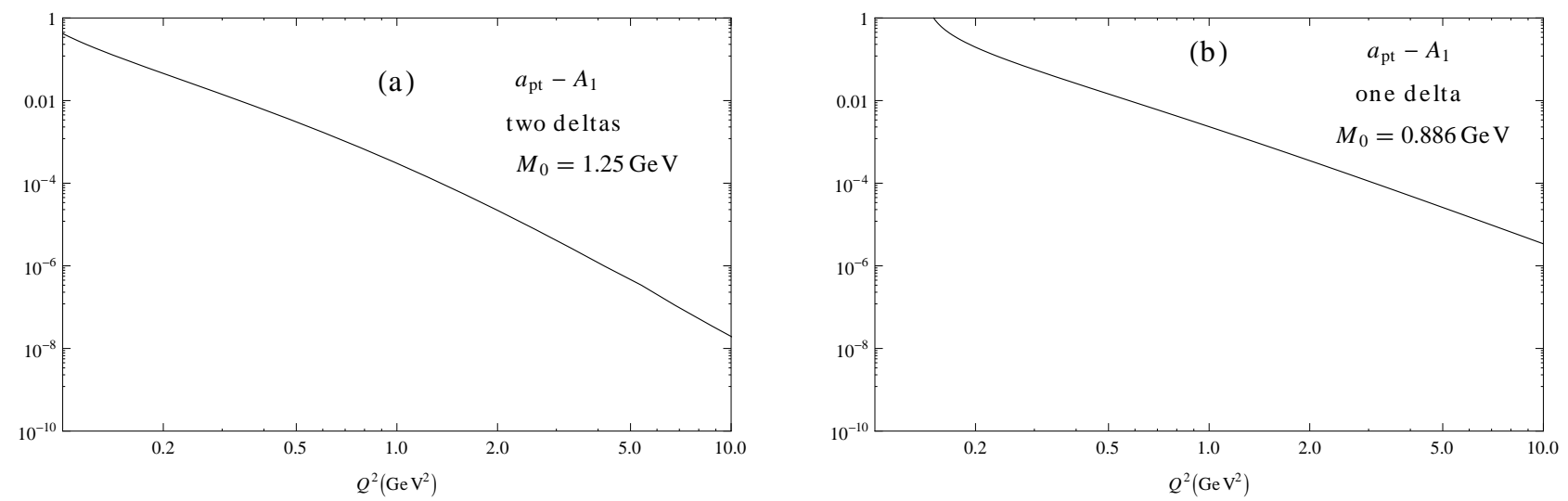

FIG. 6: The difference between the perturbative and the analytic coupling, as a function of positive $Q^{2}$, (a) the considered two-delta anQCD model (with $M_{0}=1.25 \mathrm{GeV}$ ); (b) for the one-delta anQCD parameter of Ref. [26], but with the input parameters somewhat modified (see the text for details).

the difference $\left(a_{\mathrm{pt}}-\mathcal{A}_{1}\right)$ in the case of the one-delta anQCD model of [26], with parameters as given in the last line of Table \.

From Fig. 6(a), we can deduce that the difference $\left(a_{\mathrm{pt}}-\mathcal{A}_{1}\right)$ behaves as $\propto\left(\Lambda^{2} / Q^{2}\right)^{n_{\text {eff }}}$, where the numerical value of the effective power index $n_{\text {eff }}$ is somewhat less than 5 ; it is somewhere between 4 and 5 . This can be understood in the following way: the values of $s_{0}$ and $s_{1}$ in the model are relatively large $\left(\sim 10^{1}\right)$, and therefore the coefficients $\mathcal{K}_{n}($ for $n=5,6, \ldots)$ in the expansion

$$
a_{\mathrm{pt}}\left(Q^{2} ; c_{2}\right)-\mathcal{A}_{1}\left(Q^{2} ; c_{2}\right)=\mathcal{K}_{5}\left(\Lambda^{2} / Q^{2}\right)^{5}+\mathcal{K}_{6}\left(\Lambda^{2} / Q^{2}\right)^{6}+\ldots
$$

are large $\left(\sim s_{0}^{n-1}, s_{1}^{n-1}\right)$ and are increasing when $n$ increases.

Finally, in Table [I] we compare the convergence of the series for $r_{\tau}$, Eq. (43), in the three cases of the two-delta model and in the one-delta model of Table I. We see that the convergence is good in general, and appears to be better when the underlying pQCD scheme parameter $c_{2}$ is more negative.

There is one more aspect of the presented model that we should address. We recall in more detail how we matched the presented anQCD model indirectly to the $\overline{\mathrm{MS}}$ scheme, via the condition (19): 
TABLE II: The four terms in the truncated analytic expansion (43) in two-delta anQCD model for the three choices of the pQCD-onset scale $M_{0}$ and for the one-delta anQCD model, as specified in Table 【]

\begin{tabular}{|c|c|c|c|c|c|c|c|}
\hline$M_{0}[\mathrm{GeV}]$ & $c_{2}$ & $\overline{\Lambda[\mathrm{GeV}]}$ & $r_{\tau}: \mathrm{LB}$ & NLB & $\mathrm{N}^{2} \mathrm{LB}$ & $\mathrm{N}^{3} \mathrm{LB}$ & $\overline{\text { sum (sum) }}$ \\
\hline 1.00 & -7.15 & 0.193 & 0.2023 & 0.0012 & -0.0010 & 0.0005 & 0.2030 \\
\hline 1.25 & -4.76 & 0.260 & 0.2052 & 0.0013 & -0.0053 & 0.0019 & 0.2030 \\
\hline 1.50 & -2.10 & 0.363 & 0.2096 & 0.0013 & -0.0110 & 0.0030 & 0.2030 \\
\hline 0.886 & 0 & 0.472 & 0.2149 & 0.0014 & -0.0176 & 0.0043 & 0.2030 \\
\hline
\end{tabular}

1. The Lambert scale of the model, at $n_{f}=3$, was chosen to coincide with the Lambert scale of the underlying pQCD model in the same scheme determined by the beta function of Eq. (10) at $n_{f}=3$;

2. The latter scale was fixed in such a way that the change of the scheme parameters $\left(c_{2}, c_{2}^{2} / c_{1}, c_{2}^{3} / c_{1}^{2}, \ldots\right)$ to the four-loop $\overline{\mathrm{MS}}$ scheme parameters $\left(c_{2}^{\overline{\mathrm{MS}}}, c_{3}^{\overline{\mathrm{MS}}}\right)$, at $n_{f}=3$ and $\mu^{2}=\left(2 m_{c}\right)^{2}$, gave the value of $a_{\mathrm{pt}}^{(\overline{\mathrm{MS}})}\left(\left(2 m_{c}\right)^{2} ; n_{f}=3\right)$ which corresponds to $a_{\mathrm{pt}}^{(\overline{\mathrm{MS}})}\left(M_{Z}^{2}\right)=0.1184 / \pi$, the central value of the world average [33]. The RGE-running between $\mu^{2}=M_{Z}^{2}$ and $\mu^{2}=\left(2 m_{c}\right)^{2}$, in $\overline{\mathrm{MS}}$, was performed in the usual way, using the four-loop polynomial form of $\beta^{(\overline{\mathrm{MS}})}\left(a_{\mathrm{pt}}\right)$, and the three-loop matching conditions [34] at quark thresholds $\mu^{2}=\left(2 m_{q}\right)^{2}(q=b, c)$.

As pointed out in Ref. [51], the aforementioned (three-loop) matching in principle introduces, indirectly, an element of nonanalyticity in the described framework, at ${ }^{12}$ the scale $Q^{2}=\left(2 m_{c}\right)^{2}$ and (to a much lesser degree) at $Q^{2}=\left(2 m_{b}\right)^{2}$. This is so because the matching introduces nonanalyticity (even: discontinuity) in the running coupling $a_{\mathrm{pt}}^{(\overline{\mathrm{MS}})}\left(Q^{2}\right)$ at those threshold scales. It would be more convenient for the presented low-energy anQCD model to be matched [at energies $Q^{2} \sim\left(2 m_{c}\right)^{2}$ ] to a scheme which introduces the quark mass threshold effects in the running in a gradual (analytic) way. One such scheme is the pinch technique (PT), Refs. 52]. The PT effective charge (i.e., running coupling) was presented for supersymmetric QCD at one-loop in Ref. 51]. Specifically, in nonsupersymmetric QCD with three massless flavors, the relations of Ref. [51] (especially their Eq. (A3)) imply $\left(\right.$ for $\left.\left|Q_{0}^{2}\right|,\left|Q^{2}\right| \lesssim\left(2 m_{c}\right)^{2}\right)$

$$
a_{\mathrm{PT}}\left(Q^{2}\right)=a_{\mathrm{PT}}\left(Q_{0}^{2}\right)+a_{\mathrm{PT}}^{2}\left(Q_{0}^{2}\right)\left[-\frac{9}{4} \ln \left(Q^{2} / Q_{0}^{2}\right)+\frac{1}{6} L_{1 / 2}\left(Q^{2} / m_{c}^{2}\right)-\frac{1}{6} L_{1 / 2}\left(Q_{0}^{2} / m_{c}^{2}\right)\right],
$$

where the quark threshold function $L_{1 / 2}$ is

$$
L_{1 / 2}\left(Q^{2} / m^{2}\right)=\left(3-\beta^{2}\right)[\beta \operatorname{ArcTanh}(1 / \beta)-1]+2
$$

where $\beta=\sqrt{1+4 m^{2} / Q^{2}}$ and the (complex) momenta $Q^{2}$ are not on the cut $Q^{2}<-4 m^{2}$. In the case of decoupling $\left(\left|Q^{2}\right| \ll m^{2}\right)$, this function acquires the value of $(5 / 3)$.

We can estimate the practical errors introduced in the calculations in our model due to the aforementioned matching to $\overline{\mathrm{MS}}$, by estimating the errors that the actual nondecoupling of the charm quark mass introduced in our calculation of $r_{\tau}$ (note that in our calculation, we considered $c$ quark to be completely decoupled, in the spirit of $\overline{\mathrm{MS}}$ ). The relation (49) would be transcribed in our model, approximately, in the following way:

$$
\mathcal{A}_{1}\left(Q^{2}\right)_{\mathrm{thr}} \approx \mathcal{A}_{1}\left(Q_{0}^{2}\right)_{\mathrm{thr}}+\widetilde{\mathcal{A}}_{2}\left(\bar{Q}^{2}\right)\left[-\frac{9}{4} \ln \left(Q^{2} / Q_{0}^{2}\right)+\frac{1}{6} L_{1 / 2}\left(Q^{2} / m_{c}^{2}\right)-\frac{1}{6} L_{1 / 2}\left(Q_{0}^{2} / m_{c}^{2}\right)\right],
$$

where $\widetilde{\mathcal{A}}_{2}\left(\bar{Q}^{2}\right)$ is the logarithmic derivative of $A_{1}$ defined via Eq. (36), and the scale $\bar{Q}^{2}$ is taken to be the geometric mean ${ }^{13}$ of $Q_{0}^{2}$ and $Q^{2}: \bar{Q}^{2}=\sqrt{Q_{0}^{2} Q^{2}}$. Further, the subscript "thr" indicates that the nondecoupling effect $m_{c} \neq \infty$ is taken into account, in a first approximation. The corresponding relation in the model without the analytic threshold effects is

$$
\mathcal{A}_{1}\left(Q^{2}\right) \approx \mathcal{A}_{1}\left(Q_{0}^{2}\right)+\widetilde{\mathcal{A}}_{2}\left(\bar{Q}^{2}\right)\left[-\frac{9}{4} \ln \left(Q^{2} / Q_{0}^{2}\right)\right]
$$

12 We consider, throughout, the first three flavors to be massless.

13 Formally, at one-loop level, it would be equivalent to take the scale $\bar{Q}^{2}=Q_{0}^{2}$ or $\bar{Q}^{2}=Q^{2}$; numerically, though, the geometric mean of these two scales (i.e., the arithmetic mean of the logarithms of these scales) is better. 
TABLE III: The estimates of nondecoupling of $m_{c}$ in the calculation of $r_{\tau}$, Eq. (54), for different value of the parameter $\kappa$. In the third column we include a measure of the effect of nondecoupling of $m_{c}$ at $Q_{0}^{2}=\kappa m_{\tau}^{2}$ (assumed to be negligible in Eq. (54)); and in the fourth column the ratio of $\frac{9}{4} \ln \left(Q^{2} / Q_{0}^{2}\right) \widetilde{\mathcal{A}}_{2}\left(\bar{Q}^{2}\right)$ with $\mathcal{A}_{1}\left(Q_{0}^{2}\right)$ (assumed to be small in (51) and (52)), for $Q^{2}=m_{\tau}^{2}$ and $Q_{0}^{2}=\kappa m_{\tau}^{2}$.

\begin{tabular}{llll}
\hline \hline$\kappa$ & $\delta r_{\tau}^{(\mathrm{LO})}\left(m_{c} \neq \infty\right)$ & $\left(L_{1 / 2}\left(\kappa m_{\tau}^{2} / m_{c}^{2}\right)-L_{1 / 2}(0)\right) / L_{1 / 2}(0)$ & $-\frac{9}{4} \ln (\kappa) \widetilde{\mathcal{A}}_{2}\left(\sqrt{\kappa} m_{\tau}^{2}\right) / \mathcal{A}_{1}\left(\kappa m_{\tau}^{2}\right)$ \\
\hline 0.15 & $6.1 \times 10^{-4}$ & 0.034 & 0.42 \\
0.20 & $4.6 \times 10^{-4}$ & 0.045 & 0.36 \\
0.30 & $3.1 \times 10^{-4}$ & 0.066 & 0.28 \\
0.40 & $2.3 \times 10^{-4}$ & 0.087 & 0.21 \\
\hline \hline
\end{tabular}

An estimate of the error in the calculation of $r_{\tau}$, due to the nondecoupling of $m_{c}$, in the calculation of $r_{\tau}$ can be obtained by using the relation (37) in the leading order (LO) when $d_{\text {Adl }}\left(Q^{2}\right)=\mathcal{A}_{1}\left(Q^{2}\right)$ there, and subtracting for $\mathcal{A}_{1}\left(Q^{2}\right)$ the two relations (51) and (52) on the contour

$$
\begin{aligned}
\delta r_{\tau}^{(\mathrm{LO})}\left(m_{c} \neq \infty\right)= & \left.\frac{1}{2 \pi} \int_{-\pi}^{+\pi} d \phi\left(1+e^{i \phi}\right)^{3}\left(1-e^{i \phi}\right)\left[\mathcal{A}_{1}\left(Q^{2}\right) \mathrm{thr}-\mathcal{A}_{1}\left(Q^{2}\right)\right]\right|_{Q^{2}=m_{\tau}^{2} e^{i \phi}} \\
\approx & \frac{1}{2 \pi} \int_{-\pi}^{+\pi} d \phi\left(1+e^{i \phi}\right)^{3}\left(1-e^{i \phi}\right) \\
& \times\left.\left[\left(\mathcal{A}_{1}\left(Q_{0}^{2}\right)_{\mathrm{thr}}-\mathcal{A}_{1}\left(Q_{0}^{2}\right)\right)+\frac{1}{6} \widetilde{\mathcal{A}}_{2}\left(\sqrt{Q_{0}^{2} Q^{2}}\right)\left(L_{1 / 2}\left(Q^{2} / m_{c}^{2}\right)-L_{1 / 2}\left(Q_{0}^{2} / m_{c}^{2}\right)\right)\right]\right|_{Q^{2}=m_{\tau}^{2} e^{i \phi}} .
\end{aligned}
$$

The scale $Q_{0}^{2}$ here should have a low value $\left(\left|Q_{0}^{2}\right| \ll m_{\tau}^{2}\right)$ so that the charm quark can be assumed to basically decouple $\left(m_{c}=\infty\right)$ at such scale. Namely, in such a case, the first (unknown) term in the integrand, $\left(\mathcal{A}_{1}\left(Q_{0}^{2}\right)_{\mathrm{thr}}-\mathcal{A}_{1}\left(Q_{0}^{2}\right)\right)$, can be taken to be zero, and the remaining term would indicate correctly the effects of nondecoupling of $m_{c}$. On the other hand, $\left|Q_{0}\right|^{2}$ cannot be taken to be too small, as then the $\sim \widetilde{\mathcal{A}}_{2}$ term in the relations (51) and (52) would dominate over the $\mathcal{A}_{1}\left(Q_{0}^{2}\right)$ term, due to a very large value of the logarithm $\ln \left(Q^{2} / Q_{0}^{2}\right)$. Therefore, we will choose $Q_{0}^{2}=\kappa Q^{2}\left(=\kappa m_{\tau}^{2} \exp (i \phi)\right)$, with $\kappa<1$ varying in a specific interval were both aforementioned restrictions are reasonably fulfilled

$$
\left.\delta r_{\tau}^{(\mathrm{LO})}\left(m_{c} \neq \infty\right) \approx \frac{1}{12 \pi} \int_{-\pi}^{+\pi} d \phi\left(1+e^{i \phi}\right)^{3}\left(1-e^{i \phi}\right) \widetilde{\mathcal{A}}_{2}\left(\sqrt{Q_{0}^{2} Q^{2}}\right)\left(L_{1 / 2}\left(Q^{2} / m_{c}^{2}\right)-L_{1 / 2}\left(Q_{0}^{2} / m_{c}^{2}\right)\right)\right|_{Q^{2}=m_{\tau}^{2} e^{i \phi} ; Q_{0}^{2}=\kappa Q^{2}} .
$$

The results of these estimates, for the central value of the parameters of the presented two-delta anQCD model (i.e., the case $M_{0}=1.25 \mathrm{GeV}$ of Table II) are presented in Table [II]. We see from the Table that the effects of the introduction of analytic threshold effects (i.e., the effects of the nondecoupling of $m_{c}$ ) in the calculation of $r_{\tau}$ are $\delta r_{\tau} \lesssim 10^{-3}$. This is to be compared with the theoretical value $r_{\tau}^{(\mathrm{LO})} \approx 0.123$ in the anQCD model, and the full value $r_{\tau}=0.203$ in the model, and with the experimental uncertainties $\delta r_{\tau}= \pm 0.004$ [Eq. (23)]. We conclude that, although these effects are appreciable, they are somewhat lower than the present experimental uncertainty of $r_{\tau}$.

\section{SUMMARY}

In this work we presented an analytic QCD (anQCD) model which, at high squared momenta $\left|Q^{2}\right|$, becomes to a high degree indistinguishable from perturbative QCD (pQCD); nonetheless, at low $\left|Q^{2}\right|$ the spacelike couplings $\mathcal{A}_{n}\left(Q^{2}\right)$ of the model mirror correctly the analytic properties of the (to-be-evaluated) spacelike observables $\mathcal{D}\left(Q^{2}\right)$ as dictated by the general principles of the local quantum field theories. The model reproduces correctly the experimental value of the $\tau$ lepton (nonstrange, $V+A$ ) semihadronic decay ratio $r_{\tau}$, i.e., the only low-momentum QCD observable that is well measured and whose higher-twist effects are small and appear to be under control. The difference between the analytic $\mathcal{A}_{1}\left(Q^{2}\right)$ and its perturbative counterpart $a_{\mathrm{pt}}\left(Q^{2}\right)\left[\equiv \alpha_{s}\left(Q^{2}\right) / \pi\right]$ is formally $\sim\left(\Lambda^{2} / Q^{2}\right)^{5}$ at $\left|Q^{2}\right|>\Lambda^{2}$, where $\Lambda^{2} \sim 0.1 \mathrm{GeV}^{2}$ is the QCD (or: light meson) scale. The starting point was the construction of the discontinuity function $\rho_{1}(\sigma)$ for the analytic coupling $\mathcal{A}_{1}\left(Q^{2}\right)$. This $\rho_{1}(\sigma)$ is assumed to coincide with its pQCD counterpart for $\sigma$ above a pQCD-onset scale $M_{0}^{2} \sim 1 \mathrm{GeV}^{2}$. On the other hand, the unknown behavior of $\rho_{1}(\sigma)$ in the low- $\sigma$ regime is parametrized as a linear combination of two delta functions. The underlying pQCD scheme parameter $c_{2}$ is adjusted so that the pQCD-onset scale $M_{0}$ is either $1.0,1.25$, or $1.50 \mathrm{GeV}$. We believe that these three variants of the two-delta anQCD model represent almost the same physics, since they reproduce the same value of the key low-momentum 
observable $r_{\tau}(V+A)$ and, at the same time, they differ in the value of the underlying pQCD (i.e., high-momentum) renormalization scheme parameter $c_{2}$, the latter being the only free input parameter of the model. If the value of $c_{2}$ is further adjusted so that $M_{0}$ is below $1.0 \mathrm{GeV}$, the analyticity gets lost at $M_{0} \approx 0.9 \mathrm{GeV}$. The model is an extension of the one-delta anQCD model of [26] where $c_{2}=0$ was used.

The main motivation behind the construction of the considered anQCD model is that with it we can eventually evaluate low-momentum QCD quantities whose higher-twist contributions are appreciable. An example are the separate vector $(V)$ and axial vector $(A)$ channel of $r_{\tau}$. Such evaluations would involve the considered anQCD model together with the Operator Product Expansion (OPE). This application (anQCD+OPE) is consistent, due to the very suppressed difference $\mathcal{A}_{1}\left(Q^{2}\right)-a_{\mathrm{pt}}\left(Q^{2}\right) \sim\left(\Lambda^{2} / Q^{2}\right)^{5}$ in the ultraviolet regime; the latter implying that the higher-twist terms in OPE, of dimension $d \leq 8$, will still be of infrared origin, in accordance with the philosophy of the ITEP (Institute of Theoretical and Experimental Physics) group [23].

\section{Acknowledgments}

This work was supported in part by FONDECYT Grant No. 1095196 (C.C., G.C.), Rings Project ACT119 (G.C.), and Mecesup2 Grant FSM 0605-D3021 (C.A.). This work was influenced by the ideas and suggestions of our colleague Olivier Espinosa (1961-2010), coauthor of our previous work Ref. [26].

[1] N.N. Bogoliubov and D.V. Shirkov, Introduction to the Theory of Quantum Fields, New York, Wiley, 1980.

[2] R. Oehme, Int. J. Mod. Phys. A 10, 1995 (1995) arXiv:hep-th/9412040.

[3] C. Lerche and L. von Smekal, Phys. Rev. D 65, 125006 (2002) hep-ph/0202194.

[4] R. Alkofer, C. S. Fischer and F. J. Llanes-Estrada, Phys. Lett. B 611, 279 (2005) [Erratum-ibid. 670, 460 (2009)] arXiv:hep-th/0412330; A. C. Aguilar, D. Binosi and J. Papavassiliou, Phys. Rev. D 78, 025010 (2008) arXiv:0802.1870 [hep-ph]]; A. C. Aguilar, D. Binosi, J. Papavassiliou and J. Rodriguez-Quintero, Phys. Rev. D 80, 085018 (2009) arXiv:0906.2633 [hep-ph]].

[5] A. Cucchieri and T. Mendes, Phys. Rev. Lett. 100, 241601 (2008) arXiv:0712.3517 [hep-lat]]; I. L. Bogolubsky, E. M. Ilgenfritz, M. Muller-Preussker and A. Sternbeck, Phys. Lett. B 676, 69 (2009) arXiv:0901.0736 [hep-lat]].

[6] D. V. Shirkov and I. L. Solovtsov, hep-ph/9604363 Phys. Rev. Lett. 79, 1209 (1997) arXiv:hep-ph/9704333.

[7] K. A. Milton, I. L. Solovtsov and O. P. Solovtsova, Phys. Lett. B 415, 104 (1997) hep-ph/9706409.

[8] D. V. Shirkov, Theor. Math. Phys. 127, 409 (2001) hep-ph/0012283; Eur. Phys. J. C 22, 331 (2001) hep-ph/0107282.

[9] A. P. Bakulev, S. V. Mikhailov and N. G. Stefanis, Phys. Rev. D 72, 074014 (2005) [Erratum-ibid. D 72, 119908 (2005)] arXiv:hep-ph/0506311]; Phys. Rev. D 75, 056005 (2007) [Erratum-ibid. D 77, 079901 (2008)] arXiv:hep-ph/0607040; JHEP 1006, 085 (2010) arXiv:1004.4125 [hep-ph]].

[10] A. V. Nesterenko, Phys. Rev. D 62, 094028 (2000) arXiv:hep-ph/9912351; Phys. Rev. D 64, 116009 (2001) arXiv:hep-ph/0102124; Int. J. Mod. Phys. A 18, 5475 (2003) arXiv:hep-ph/0308288; A. C. Aguilar, A. V. Nesterenko and J. Papavassiliou, J. Phys. G 31, 997 (2005).

[11] G. Cvetič and C. Valenzuela, J. Phys. G 32, L27 (2006) arXiv:hep-ph/0601050.

[12] G. Cvetič and C. Valenzuela, Phys. Rev. D 74, 114030 (2006) arXiv:hep-ph/0608256.

[13] A. V. Nesterenko and J. Papavassiliou, Phys. Rev. D 71, 016009 (2005);

[14] D. V. Shirkov, Nucl. Phys. Proc. Suppl. 162, 33 (2006) arXiv:hep-ph/0611048.

[15] D. V. Shirkov and I. L. Solovtsov, Theor. Math. Phys. 150, 132 (2007) arXiv:hep-ph/0611229.

[16] D. V. Shirkov, Theor. Math. Phys. 119, 438 (1999) [Teor. Mat. Fiz. 119, 55 (1999)] arXiv:hep-th/9810246]; Lett. Math. Phys. 48, 135 (1999).

[17] G. Cvetič and A. V. Kotikov, J. Phys. G 39, 065005 (2012) arXiv:1106.4275 [hep-ph]].

[18] G. Cvetič, R. Kögerler, C. Valenzuela, J. Phys. G G37, 075001 (2010) arXiv:0912.2466] [hep-ph]]; Phys. Rev. D82, 114004 (2010) arXiv:1006.4199 [hep-ph]].

[19] A. V. Nesterenko and J. Papavassiliou, J. Phys. G 32, 1025 (2006) arXiv:hep-ph/0511215; A. V. Nesterenko, Proc. of the Ninth Workshop on Non-Perturbative QCD (June 4-8, 2007, Paris, France), eConf C 0706044, 25 (2007) arXiv:0710.5878 [hep-ph]]; Proc. of the Eleventh Workshop on Nonperturbative QCD (June 6-10, 2011, Paris, France), eConf C 1106064, 23 (2011) arXiv:1106.4006 [hep-ph]].

[20] S. Peris, M. Perrottet and E. de Rafael, JHEP 9805, 011 (1998) arXiv:hep-ph/9805442.

[21] E. de Rafael, Nucl. Phys. Proc. Suppl. 96, 316 (2001) hep-ph/0010209; arXiv:1111.6162 [hep-ph].

[22] B. A. Magradze, Few Body Syst. 48, 143 (2010) arXiv:1005.2674 [hep-ph]].

[23] M. A. Shifman, A. I. Vainshtein and V. I. Zakharov, Nucl. Phys. B 147, 385 (1979); Nucl. Phys. B 147, 448 (1979).

[24] S. Peris, Phys. Rev. D 74, 054013 (2006) arXiv:hep-ph/0603190.

[25] G. Cvetič and H. E. Martínez, J. Phys. G 36, 125006 (2009) [arXiv:0907.0033 [hep-ph]].

[26] C. Contreras, G. Cvetič, O. Espinosa and H. E. Martínez, Phys. Rev. D 82, 074005 (2010) arXiv:1006.5050]. 
[27] MATHEMATICA 8.0.4, Wolfram Co.

[28] E. Gardi, G. Grunberg and M. Karliner, JHEP 9807, 007 (1998) hep-ph/9806462;

[29] D. S. Kourashev, arXiv:hep-ph/9912410 D. S. Kurashev and B. A. Magradze, Theor. Math. Phys. 135, 531 (2003) [Teor. Mat. Fiz. 135, 95 (2003)].

[30] B. A. Magradze, Few Body Syst. 40, 71 (2006) hep-ph/0512374.

[31] G. Cvetič and I. Kondrashuk, JHEP 1112, 019 (2011) arXiv:1110.2545 [hep-ph]].

[32] R. M. Corless, G. H. Gonnet, D. E. G. Hare, D. J. Jeffrey, D. E. Knuth, "On the Lambert W function," Adv. Comput. Math. 5, 329-359 (1996).

[33] K. Nakamura et al. [Particle Data Group], J. Phys. G 37, 075021 (2010).

[34] K. G. Chetyrkin, B. A. Kniehl and M. Steinhauser, Phys. Rev. Lett. 79, 2184 (1997) arXiv:hep-ph/9706430.

[35] R. Barate et al. [ALEPH Collaboration], Eur. Phys. J. C 4, 409 (1998).

[36] S. Schael et al. [ALEPH Collaboration], Phys. Rept. 421, 191 (2005) arXiv:hep-ex/0506072].

[37] K. Ackerstaff et al. [OPAL Collaboration], Eur. Phys. J. C 7, 571 (1999) arXiv:hep-ex/9808019].

[38] M. Davier, S. Descotes-Genon, A. Höcker, B. Malaescu and Z. Zhang, Eur. Phys. J. C 56, 305 (2008) arXiv:0803.0979 [hep-ph]].

[39] B. L. Ioffe, Prog. Part. Nucl. Phys. 56, 232 (2006) arXiv:hep-ph/0502148].

[40] K. Maltman and T. Yavin, Phys. Rev. D 78, 094020 (2008) arXiv:0807.0650 [hep-ph]].

[41] E. Braaten, Phys. Rev. Lett. 60, 1606 (1988); S. Narison and A. Pich, Phys. Lett. B 211, 183 (1988); E. Braaten, S. Narison, and A. Pich, Nucl. Phys. B 373, 581 (1992); A. Pich and J. Prades, JHEP9806, 013 (1998) hep-ph/9804462.

[42] K. G. Chetyrkin, A. L. Kataev and F. V. Tkachov, Phys. Lett. B 85, 277 (1979); M. Dine and J. R. Sapirstein, Phys. Rev. Lett. 43, 668 (1979); W. Celmaster and R. J. Gonsalves, Phys. Rev. Lett. 44, 560 (1980).

[43] S. G. Gorishnii, A. L. Kataev and S. A. Larin, Phys. Lett. B 259, 144 (1991); L. R. Surguladze and M. A. Samuel, Phys. Rev. Lett. 66, 560 (1991) [Erratum-ibid. 66, 2416 (1991)].

[44] P. A. Baikov, K. G. Chetyrkin and J. H. Kühn, Phys. Rev. Lett. 101, 012002 (2008) arXiv:0801.1821 [hep-ph]].

[45] D. J. Broadhurst, Z. Phys. C 58, 339 (1993).

[46] M. Beneke, Phys. Lett. B 307, 154 (1993); Nucl. Phys. B 405, 424 (1993).

[47] M. Neubert, Phys. Rev. D 51, 5924 (1995) hep-ph/9412265;

[48] M. Neubert, hep-ph/9502264.

[49] C. Amsler et al. [Particle Data Group], Phys. Lett. B 667, 1 (2008).

[50] A. I. Alekseev, Few Body Syst. 40, 57 (2006) arXiv:hep-ph/0503242.

[51] M. Binger and S. J. Brodsky, Phys. Rev. D 69, 095007 (2004) hep-ph/0310322.

[52] J. M. Cornwall, Phys. Rev. D 26, 1453 (1982); G. Degrassi and A. Sirlin, Phys. Rev. D 46, 3104 (1992); N. J. Watson, Nucl. Phys. B 494, 388 (1997) hep-ph/9606381; J. Papavassiliou, E. de Rafael and N. J. Watson, Nucl. Phys. B 503, 79 (1997) hep-ph/9612237]; D. Binosi, J. Phys. G G 30, 1021 (2004) hep-ph/0401182. 